\title{
Modeling and Analyzing Operational Decision-Making Synchronization of C2 Organization in Complex Environment
}

\author{
Zou Zhigang, Liu Fuxian, Zhang Bo, and Li Xiang \\ Air and Missile Defense College, Air Force Engineering University, Xian 710051, China \\ Correspondence should be addressed to Zou Zhigang; usher12@126.com
}

Received 5 June 2013; Revised 24 July 2013; Accepted 24 July 2013

Academic Editor: J. A. Tenreiro Machado

Copyright ( 2013 Zou Zhigang et al. This is an open access article distributed under the Creative Commons Attribution License, which permits unrestricted use, distribution, and reproduction in any medium, provided the original work is properly cited.

\begin{abstract}
In order to improve capability of operational decision-making synchronization (ODMS) in command and control (C2) organization, the paper puts forward that ODMS is the negotiation process of situation cognition with three phases about "situation cognition, situation interaction and decision-making synchronization" in complex environment, and then the model and strategies of ODMS are given in quantity. Firstly, measure indexes of three steps above are given in the paper based on the time consumed in negotiation, and three patterns are proposed for negotiating timely in high quality during situation interaction. Secondly, the ODMS model with two stages in continuous changing situation is put forward in the paper, and ODMS strategies are analyzed within environment influence and time restriction. Thirdly, simulation cases are given to validate the process of ODMS under different continuous changing situations the results of this model are better than the other previous models to fulfill the actual restrictions, and the process of ODMS can be adjusted more reasonable for improving the capability of ODMS. Then we discuss the case and summarize the influence factors of ODMS in the C2 organization as organization structure, shared information resources, negotiation patterns, and allocation of decision rights.
\end{abstract}

\section{Introduction}

As remarkable characteristics of joint operation under information condition, systems combat demands that operational entities should form the coincident combat intention within networked operational systems in cognition domain, and then the entities can be driven to take cooperative action in physical domain, which makes operation synchronization realized to improve combat effectiveness of weapons systems in the furthest extent [1]. So study in operation synchronization has great significance on combat effectiveness of networked operational systems.

In the concept of network centric warfare (NCW), operation synchronization is mainly referred to self-synchronization [2]. As the development of NCW, the concept of network centric operations (NCO) is paid more attention to decisionmaking synchronization and action synchronization. Based on situation awareness within complex environment in information domain, situation cognition difference can be formed within different operation entities, respectively. Then certain regulations or strategies should be followed by each operation entity to realize situation interaction in high quality, which can achieve operational decision-making synchronization (ODMS) with unanimous situation cognition [3]. Then different operational entities are driven to take cooperative action within operational systems in physical domain. So in order to study operation synchronization in quantity, the primary question about how to model and improve ODMS should be solved firstly.

At present, the qualitative expatiation about ODMS is the essential requirement for networked systems combat in the future is put forward in $[2,3]$. In order to study ODMS in quantity, ODMS is taken into account as complex activity in cognition domain within the command and control (C2) organization, and then some measure indexes and models of ODMS are given on the basis of C2 organization with mainly three methods as follows.

The first method is based on opinion emergence of group communication [4], ODMS is regarded as opinion exchange process on the basis of group communication in $[5,6]$. There are two shortcomings as follows: (1) communication is the process of exchange opinion among individuals but not 
always obtain the consensus in the whole organization [7]. The illogical strategies and ambiguous indexes of communication are difficult to be used form the unanimous opinions directly, but mostly in oppose opinions, multiple opinions, and so on. (2) In actual combat process, operation plans can be formed by operation entities with situation cognition, respectively, and then ODMS can be is realized by operation plan-based negotiation among operation entities, which is named as situation interaction [8]. However, situation cognition is abstract and time variable in cognition domain. Presently as improvement on "three-layer" model of situation awareness $[9,10]$, the researches on situation cognition, such as individual situation awareness [11], and situation awareness velocity [12], are deficient in analyzing situation cognition formation, and the research about influence of complex changing environment on the ODMS is still in blank.

The second method is with the macroscopic viewpoint of difference in situation cognition, models about cognitiveentropy (CE) and cognitive self-synchronization (CSSync) are put forward by Manso and Moffat in the American department of defense (DoD) [13], and the compute method is given on the basis of the Experimental Laboratory for Investigating Collaboration, Information-sharing and Trust (ELICIT) [14]. The models are also not referred to situation cognition formation, especially cognition interaction process, and the relation between the CE and information entropy.

The third method is that ODMS model can be constructed as network dynamic as physics systems with the microscopic viewpoint of individual cognition. Dekker in Australian national defense research centre considers each operation entity as a coupled oscillator with different phase angles [15]. As an abstract model for synchronization in C2 organization, Kuramoto model is extended to describing the ODMS [16]. Information flow (including sensor information, operation scheme, and so on) in the organization is synthesized as a phase angle, and information difference is considered as feedback of phase angle; the synchronization process can be controlled by adjusting coupled coefficients. Although the model can be used to depict the synchronization process, it excessively depends on the network synchronization in physics, which is hardly applied to actual military operation.

The ODMS demands situation interaction among operation entities to achieve unanimous situation cognition, and situation interaction can be adjusted with different patterns agilely but only certain coefficients. Therefore, combining macroscopic view and microscopic view, this paper presents that ODMS is a negotiation process of situation cognition interaction in complex environment, which contains three phases about "situation cognition, situation interaction and decision-making synchronization."

Taking time consumed into account, we construct the ODMS model with measure indexes of three phases in the paper. Then ODMS strategies are analyzed to adapt the environment influence and time restriction. Finally, the process of ODMS is verified in simulation cases in complex environment, including continuous changing situation, environment influence, and time restriction, which reflects that the model and strategies are feasible and available. On the basis of this, via analyzing and discussing the simulation results, the ODMS inhibitors and enablers are summarized in the paper.

The paper is organized as follows: measure indexes of ODMS in complex environment are given in Section 2; ODMS model is constructed in Section 3, and ODMS strategies within the environment influence and time restriction are put forward in Section 4. And the simulation cases are designed to validate the model and strategies in Section 5. After comparing and discussing the simulation results, influence factors of ODMS are given in Section 6, and the conclusion and further work are given in Section 7.

\section{Measure Indexes of ODMS in Complex Environment}

The influence of complex environment on ODMS mainly contains two aspects: continuous changing situation and environment influence on communication. According to the process of ODMS with three phases in Figure 1, time-oriented measure indexes in each phase are given combining topological structure of C2 organization in the paper; especially different situation changing patterns and negotiation patterns are put forward in situation interaction stage.

2.1. Indexes of Continuous Changing Situation. Situation change can be reflected as the number change of operation entities with updating situation information rapidly. If situation changes quickly, it will arouse the action of updating situation information within sufficient limit time. So situation information elements apperceived item by item in operational entities can be regarded as situation information flow and depicted as continuous changing functions.

Definition 1. Situation change rate (SCR): it is the ratio of the number of operational entities with updating situation information, brought by the situation change directly in unit time, to the total number of operational entities in $\mathrm{C} 2$ organization, marked as $h_{t}^{\prime}$ in formula (1), which can be used to reflect the speed of situation change:

$$
h_{t}^{\prime}=\frac{n_{t}}{N}
$$

where $n_{t} \in[0, N]$ denotes that number of operational entities with updating situation information in the moment of $t, N$ is the total number of operational entities in the C2 organization, and $h_{t}^{\prime} \in[0,1]$ shows the SCR in the time of $t$. Combined [12], three patterns of continuous changing situation are put forward in Figure 2, whose general expressions are given in Table 1.

In Table $1, a \in[0,1], b>0$, and if $a=0$, then the 1st pattern $h_{t}^{\prime}=0$ reflects that there is no operational entity updating the situation information in the time of $t$, which shows that the situation is stable (as one special case of continuous changing situation). Via compound calculation with the three patterns of continuous changing situation, more complex patterns of situations change can be generated in the paper. 


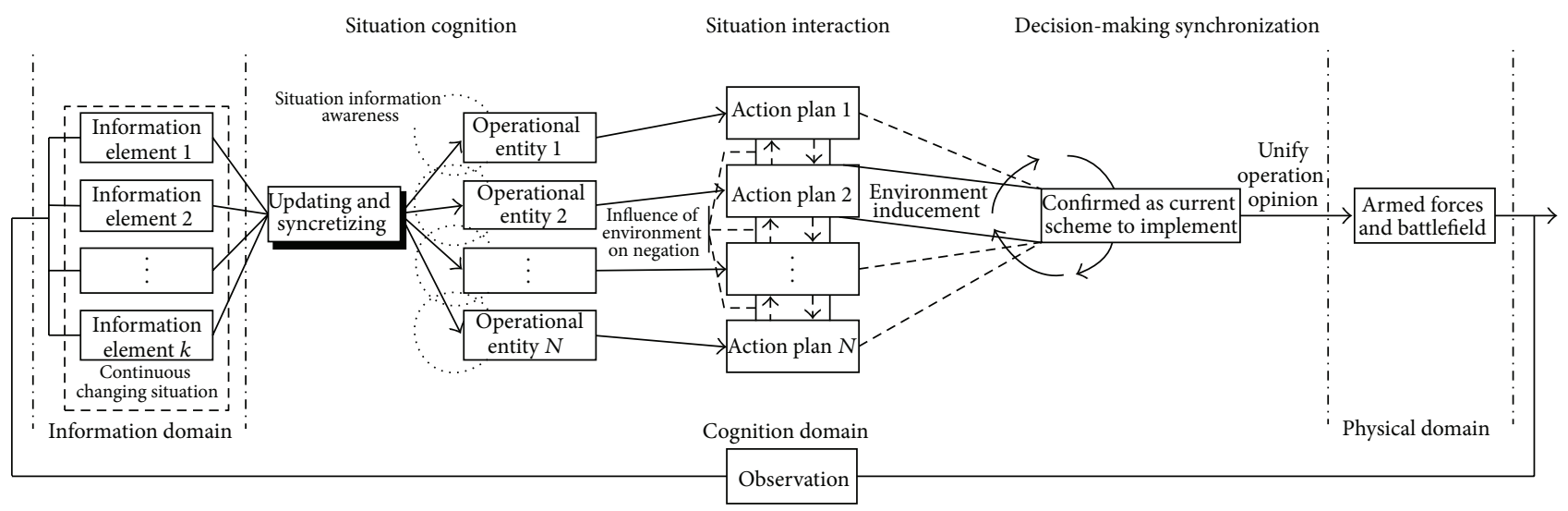

FIGURE 1: The process of ODMS in complex environment.

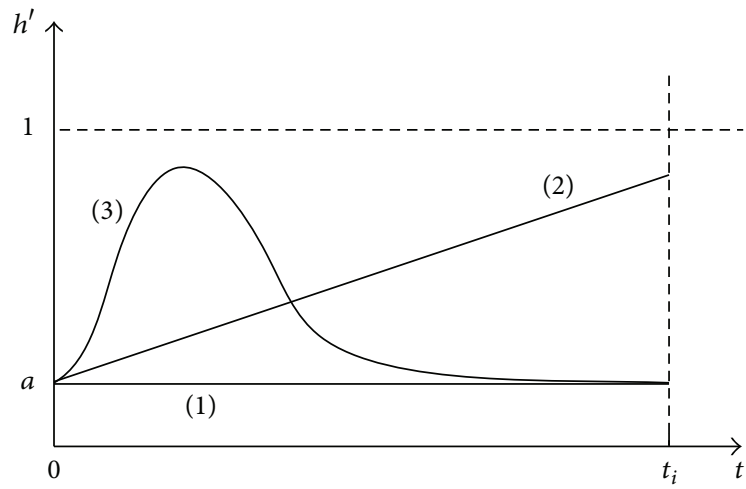

FIGURE 2: Three patterns in continuous changing situation.

2.2. Indexes of Situation Cognition. During the situation changing process, different situation cognition can be formed within different operational entities [10]. Due to the limits of time and resources, each operational entity can hardly negotiate with all the others. In the actual combat process, operational entity should negotiate with the surroundings. Taking the situation cognition level of certain operational entity (such as superior command center) as target, the others should adjust their own situation cognition via negotiating with the operational entity rapidly, and the ODMS in C2 organization can be realized when the situation cognition of others is consistent with target entity. Then indexes of situation cognition are defined as follows.

Definition 2. Situation cognition level (SCL): it is the maturity degree about operational entities in analyzing and comprehending the situation information of battlefield to form the action plans. As the improvement of discrete model in situation cognition in [17], SCL can be used to describe continuous process of individual situation cognition and marked as $x_{i} \in[0,1]$. When $x_{i}=0$ denotes that SCL of operational entity $i$ is in the blank status, $x_{i}=1$ shows that the SCL of operational entity $i$ is in the complete status, and $x_{i} \in(0,1)$ shows that the SCL of operational entity $i$ is in the forming status.
TABLE 1: The SCR in three patterns.

\begin{tabular}{llc}
\hline No. & Situation changing patterns & SCR \\
\hline 1 & Identical changing situation & $h_{t}^{\prime}=a$ \\
2 & Gradual changing situation & $h_{t}^{\prime}=b * t+a$ \\
3 & Sudden changing situation & $h_{t}^{\prime}=f(t)$ \\
\hline
\end{tabular}

Definition 3. Situation cognition target (SCT): in situation interaction process, taking the SCL of operational entity $j$ as target, different patterns can be chosen by operational entity $i$ to negotiate with $j$ for improving its SCL. Thus operational entity $i$ is called as compromise entity, and operational entity $j$ is called as target entity, and $x_{j}$ is named as SCT.

Definition 4. Information perception ability (IPA): in the process of situation cognition formation, it is used to reflect the ability of analyzing and comprehending situation information in operational entity $i$, marked as $v_{i}$. With the time of operational entity $i$ analyzing the situation information increasing, the SCL can be continuous improved. The more network resources are possessed by the operational entity in the C2 organization, the higher value of its IPA [18]; and the higher network average degree of $\mathrm{C} 2$ organization, the stronger the degree of the whole IPA:

$$
v_{i}=e^{\alpha} \cdot k_{i}^{\left\langle k-h_{t_{i}}^{\prime}\right\rangle} t_{i}^{\prime}
$$

where $k_{i} \in(0,1)$ is entity importance degree (EID) of the $i$ th object in C2 organization, $t_{i}^{\prime}$ is the time of situation cognition formation, and $\langle k\rangle$ denotes that network average degree of $\mathrm{C} 2$ organization, which is directly proportional with networked level. And $\alpha \in\{0,1\}$ is environment inducement factor if $\alpha=$ 1 shows that operational entity $i$ is induced as target entity, and $\alpha=0$ denotes that operational entity $i$ can be regarded as compromise entity.

2.3. Indexes of Situation Interaction. According to measure indexes of centrality in social network [19], the situation interaction is described as action plan-based negotiation in the view of time consuming. Based on the topological 
TABLE 2: The value of $\beta$ in electromagnetic environment.

\begin{tabular}{lc}
\hline Electromagnetic environment classification & $\beta$ \\
\hline Level I (simple) & $0 \sim 0.20$ \\
Level II (mild complexity) & $0.20 \sim 0.55$ \\
Level III (moderate complexity) & $0.55 \sim 0.80$ \\
Level IV (severe complexity) & $0.80 \sim 1.0$ \\
\hline
\end{tabular}

structure of C2 organization, if entity $i$ makes compromise with $j$, situation interaction route is marked as $r=\{(j, i) \mid$ $j \rightarrow i\}$, where $(j, i)$ denotes one segment in the situation interaction, and $j \rightarrow i$ shows that route of situation interaction is from $j$ to $i$. Here we give measure indexes of situation interaction as follows.

Definition 5. Information share ability (ISA): it is information share speed among operational entities, which reflects difference of negotiation speed among the operational entities. And $v_{i j}$ is marked as the ISA between operational entity $i$ and $j$. The ISA of each entity is directly proportional to EID [20]:

$$
v_{i j}=C_{i j} \cdot \frac{k_{i}+k_{j}}{2},
$$

where $k_{i}$ and $k_{j}$ are the EID of operational entity $i$ and $j$ separately, and $C_{i j} \in[0,1]$ is the communication unblocked degree. And that $C_{i j}=1-\beta_{i j}, \beta_{i j} \in[0,1]$ is environment influence factor. Then the value of $\beta$ is given in Table 2 according to the classification of electromagnetic environment in [21].

Definition 6. Situation interaction time (SIT): it is the time cost in forming the unanimous opinion between operational entities $i$ and $j$, which is marked as $t_{i j}$ :

$$
t_{i j}=\frac{\Delta x_{i j}}{v_{i j}}=\frac{x_{i}-x_{j}}{v_{i j}} .
$$

Definition 7. Situation interaction quality (SIQ): as situation interaction is realized by action plan-based negotiation, compromise extent is used to measure negotiation quality in situation interaction between operational entities $i$ and $j$, marked as $u_{i j} \in[0,1]$ in formula (5). With the SIT increasing, the SIQ is improving correspondingly; when exceeding certain time, the SIQ keeps invariability in maximum, and the SCL is in the complete status:

$$
\begin{array}{cl}
u_{i j}=0 \quad & t=0, \\
u_{i j} \in(0,1) & 0<t<t^{*}, \\
u_{i j}=1 \quad t^{*} \leq t .
\end{array}
$$

Combined EID and directions of situation interaction, three negotiation patterns are put forward in the paper. And the three functions of SIQ are defined as $u_{i j}=f\left(k_{i}, k_{j}, t\right)$ in Table 3.

In Figure 3, the 1st pattern shows the equality negotiation process between two operational entities with the same EID.
TABLE 3: Three SIQ functions in the direction of $j \rightarrow i$.

\begin{tabular}{lccc}
\hline No. & $\begin{array}{c}\text { Negotiation } \\
\text { patterns }\end{array}$ & EID & SIQ functions \\
\hline 1 & Equality pattern & $k_{i}=k_{j}$ & $u_{i}=v_{i j}^{\prime} t$ \\
2 & Persuading pattern & $k_{i}>k_{j}$ & $u_{i}=\left(v_{i j}^{\prime} t\right)^{2}$ \\
3 & Concession pattern & $k_{i}<k_{j}$ & $u_{i}=\left(v_{i j}^{\prime} t\right)^{1 / 2}$ \\
\hline
\end{tabular}

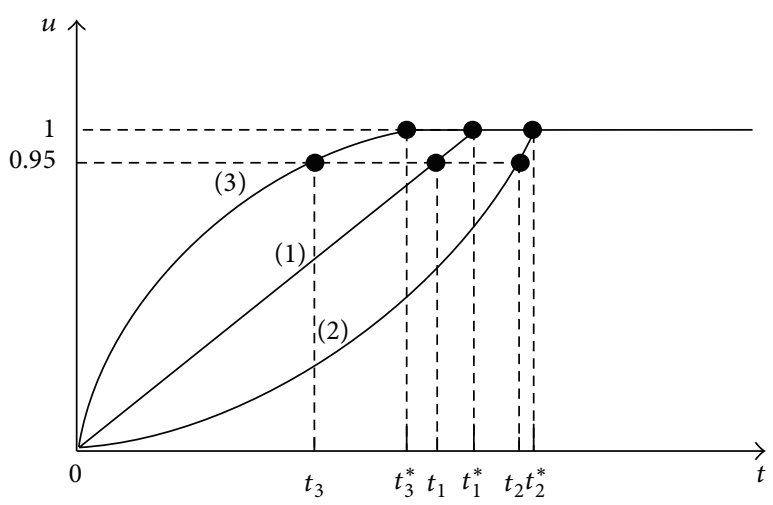

Figure 3: The SIQ of three negotiation patterns.

The 2nd pattern reflects that operational entity with high EID, as compromise entity, is persuaded to negotiate with target entity in low EID. The compromise entity is cautious to analyze and judge the SCL of target entity, as SCT, slowly in the beginning, and later gradually learn and understand from the target entity to keep the SCL consistent with SCT rapidly in a short time. The 3rd pattern denotes that operational entity with low EID as compromise entity makes a concession in negotiating with target entity in low EID. Then the compromise entity accepts the SCL of target entity as SCT directly without analysis and then learns SCT slowly after accepting in some extent to reach the SCL unanimous with target entity.

Suppose that ISA of three negotiation patterns in the direction of $j \rightarrow i$ is $v_{i j_{1} 1}, v_{i j_{2} 2}$, and $v_{i j_{3} 3}$ separately, and we can obtain that $v_{i j_{2} 2}<v_{i j_{1} 1}<v_{i j_{3} 3}$ according to formula (3). And suppose that SIT of three negotiation patterns is $t_{1}^{*}, t_{2}^{*}$, and $t_{3}^{*}$, we can gain that $t_{3}^{*}<t_{1}^{*}<t_{2}^{*}$ with formula (4). Then we can derivate relative ISA with $v_{i j}^{\prime}=v_{i j} / \Delta x_{i j}$, then $t^{*}=1 / v_{i j}^{\prime}=t_{i j}$, $0<t \leq t^{*}$. And SIQ functions are general formulas.

Definition 8. Situation interaction function: it is used to describe situation interaction process between two adjacent entities. In the direction of $j \rightarrow i, x_{i}$ can be increased by negotiating with $x_{j}$ as SCT, and can be calculated in the time of $t_{i}$ according to formula (6):

$$
x_{i}\left(t_{i}\right)=x_{i}\left(t_{i}^{\prime}\right)-u_{i}\left(t_{i}\right) \cdot\left[x_{i}\left(t_{i}^{\prime}\right)-x_{j}^{*}\right],
$$

where $x_{j}^{*}=x_{j}\left(t_{j}^{*}\right)$ denotes the SCL of operational entity $j$ after situation interaction. When $u_{i j}=0$ reflects that there is no effect in the negotiation, $u_{i j} \in(0,1)$ shows that situation interaction is accomplished in certain extent, and $u_{i j}=1$ shows that situation interaction is accomplished completely. 
2.4. Indexes of Situation Interaction. Based on certain situation prediction, SCL of someone operational entity in advantageous environment is obtained to the complete status firstly, so the operational entity is induced to be the target entity in the C2 organization. In order to improve SCL, other entities should adopt corresponding negotiation patterns to make situation interaction in high quality quickly, which can make SCL of the whole C2 organization in complete status to reach ODMS. Relative definitions are given as follows.

Definition 9. Situation prediction level (SPL): before obtaining the situation information about opposite party, original SCL of commander can be formed with active thinking and estimation in forecast process [22], which is called SPL and marked as $x_{i o}$. As network resources in C2 organization (such as the sensors and command systems) are possessed by operational entities with different EID separately, SPL of each operation entity can be generated in different extent before situation cognition formation, and $x_{i o} \geq 0$.

Definition 10. Synchronization time (ST): for each operational entity in C2 organization, it is the maximum sum of SIT and time consumed in situation cognition formation, marked as $T$ in the formula (7). After the time of $T$, the SCL of all the operational entities is in the complete status:

$$
T=\max _{i \in N}\left\{t_{i}^{\prime}+t_{i}\right\} .
$$

Definition 11. Synchronization extent (SE): it is the ratio of SCL of compromise entity $x_{i}$ after situation interaction and SCL of target entity $x_{o}$, and marked as $\gamma_{i o}$ in formula (8). Due to difference in interior condition and influence from exterior environment, ODMS is hardly accomplished completely:

$$
\gamma_{i o}=\frac{x_{i}}{x_{o}}
$$

where $\varepsilon_{i o}=1-\gamma_{i o}$ denotes accepted synchronization error. As $0 \leq x_{i}<x_{o} \leq 1, \gamma_{i o} \in[0,1]$, then $\varepsilon_{i o} \in[0,0.05]$ is defined in the paper to ensure basic ODMS and $\gamma_{i o} \in[0.95,1]$ in formula (8).

Definition 12. Synchronization time effectiveness (STE): it is the ratio of time consumed in situation cognition phase and situation interaction phase, and marked as $\tau$ in formula (9). STE reflects that time-consuming distribution of each phase in ODMS:

$$
\tau=\frac{t_{e}}{t_{r}}
$$

where $\tau>0, t_{r}=\max _{i \in N}\left\{t_{i}^{\prime}\right\}$ and $t_{e}=T-t_{e}$ is the time consuming of situation cognition and situation interaction in the $\mathrm{C} 2$ organization separately.

Definition 13. Synchronization qualities (SQ): it is the ratio of SCL increment in situation interaction phase and SCL in situation cognition phase, and it is marked as $\chi$ in formula (10). So SQ reflects the process of increasing SCL in each phase of ODMS:

$$
\chi=\frac{x_{e}}{x_{r}}
$$

where $\chi>0, x_{r}=\sum_{i \in N} x_{i}\left(t_{i}^{\prime}\right) / N$, and $x_{e}=\gamma-x_{r}$ is the average increment of SCL in phase of situation cognition and situation interaction separately.

\section{ODMS Model in Complex Environment}

3.1. Initialize C2 Organization. The triple set $\left\{G_{N}, K_{N}, X_{N}\right\}$ is used to represent $\mathrm{C} 2$ organization, $N$ is the number of operational entities, $G_{N}$ is the adjacency matrix of C2 organization, $X_{N}$ is the SPL set of operational entities, and $K_{N}=\left\{k_{i} \mid i \in N\right\}$ is the EID set of operational entities. The EID reflects the ability of operational entity possessing the network resources, which lies in the betweenness centrality and degree centrality of the entity in the C2 organization [23], and the calculation method in [24] is adopted in the paper.

3.2. Construct Model of ODMS. Decision-making synchronization experienced two phases about situation cognition and situation prediction.

Phase 1. Situation cognition: with a certain SPL, the SCL of each operational entity is increased slowly during the process of analyzing and comprehending situation information under continuous changing situation. When SCL of certain operational entity in advantageous environment is improved to the complete status in a short time, then the entity is induced to be the target entity, and other entities are regarded as compromise entities. According to formula (2), situation cognition model is constructed as follows:

$$
\begin{gathered}
x_{i}(0)=x_{i o}, \\
\dot{x}_{i}\left(t_{i}^{\prime}\right)=v_{i}\left(t_{i}^{\prime}\right)=e^{\alpha} k_{i}^{\left\langle k-h^{\prime}\left(t_{i}^{\prime}\right)\right\rangle} \cdot t_{i}^{\prime}, \\
h^{\prime}\left(t_{i}^{\prime}\right)=a \quad 0 \leq a \leq 1, \\
h^{\prime}\left(t_{i}^{\prime}\right)=b \cdot t_{i}^{\prime}+a \quad b>0, \\
h^{\prime}\left(t_{i}^{\prime}\right)=f\left(t_{i}^{\prime}\right), \quad 0 \leq h^{\prime}\left(t_{i}^{\prime}\right) \leq 1,
\end{gathered}
$$

where $i \in N, t_{i}^{\prime}$ is the time of information perception in operational entity $i$ before situation interaction, and $h^{\prime}\left(t_{i}^{\prime}\right)$ is the SCR in the time of $t_{i}^{\prime}$. When $\alpha=1$ in formula (2), the entity is regarded as target entity, and $\alpha=0$ denotes that it is compromise entity.

Phase 2. Situation interaction: based on the SCL of target entity in the complete status firstly, compromise entities make situation interaction with the target entity by applying corresponding negotiation patterns to improve their SCL in complete status, which can achieve ODMS. According to 
the formula (6), situation cognition model is constructed as follows:

$$
\begin{gathered}
S=\frac{\sum_{i=1}^{N} \gamma_{i o}}{N} \in[\gamma, 1] \\
x_{i}\left(t_{i}\right)=x_{i}\left(t_{i}^{\prime}\right)-u_{1}\left(t_{i}\right) \cdot\left[x_{i}\left(t_{i}^{\prime}\right)-x_{j}^{*}\right] \quad k_{i+1}=k_{i}, \\
x_{i}\left(t_{i}\right)=x_{i}\left(t_{i}^{\prime}\right)-u_{2}\left(t_{i}\right) \cdot\left[x_{i}\left(t_{i}^{\prime}\right)-x_{j}^{*}\right] \quad k_{i+1}=k_{i}, \\
x_{i}\left(t_{i}\right)=x_{i}\left(t_{i}^{\prime}\right)-u_{3}\left(t_{i}\right) \cdot\left[x_{i}\left(t_{i}^{\prime}\right)-x_{j}^{*}\right] \quad k_{i+1}<k_{i}, \\
0.95 \leq \gamma \leq 1, \\
(j, i) \in \Lambda=\{(m, n) \mid m \longrightarrow n, m \in P, n \in P\},
\end{gathered}
$$

where $P=\{1, \ldots, N\}$ is the set of operational entities, $u_{k}\left(t_{i}\right)(k \in\{1,2,3\})$ is the SIQ of entity $j$ applying the $k$ th negotiation pattern, and $0<t_{i} \leq t_{i}^{*}$. When $u_{i} \equiv 1$ shows the complete synchronization process and comprehensive synchronization extent as $S \equiv 1$ in formula (12) $\gamma \leq u_{i} \leq 1$ denotes the incomplete synchronization process, and $S \in$ $[0.95,1)$.

\section{ODMS Strategies within Environment and Time Restriction}

Due to influence of the environment in battlefield, such as complex environment and operation timerestrictions [21], information sharing among the operational entities in situation interaction is always incomplete, which leads to the incomplete ODMS. We suppose that situation interaction route in $\mathrm{C} 2$ organization is marked as $r=\left\{\left(p_{i}, p_{i+1}\right)\right.$ | $\left.p_{i} \rightarrow p_{i+1}, p_{i} \in G_{N}, i \in[1, N-1]\right\}$. Taken situation interaction route $j \rightarrow k \rightarrow l \cdots m$, contains $n$ situation interaction segments, as an example, the properties of ODMS are analyzed as follows in detail.

\subsection{ODMS Strategies within Environment Influence}

Property 1 (environment adaptability in choosing situation interaction route). Suppose that there are $s$ situation interaction routes existing from entity $j$ to entity $m: r_{j \rightarrow m}^{s}=$ $\left\{\left(p_{i}, p_{i+1}\right) \mid p_{i} \rightarrow p_{i+1} ; p_{i}, p_{i+1} \in G_{N} \wedge\left(p_{i}^{q}=j, p_{i+1}^{z}=m\right)\right\}$. As differences in environment influence, it leads to difference in communication unblocked degree among different interaction routes. In order to realize unblocked interaction route by any possibility, environmental adaptability operator (EAO) is designed below to choose the best situation interaction route $r_{s^{*}}$ with the minimum value, which is in favor of adapting the environment change:

$$
\Delta_{r \mid j \rightarrow m}^{s^{*}}=\min \left[\left(\sum_{i \in n} \beta_{i, i+1}-\prod_{i \in n} \beta_{i, i+1}\right) \cdot \prod_{i \in n}\left(k_{i}+k_{i+1}\right)\right] .
$$

And $\beta_{i, i+1}$ is the communication unblocked degree within the situation interaction segment $p_{i} \rightarrow p_{i+1}$.
Proof. According to recurrence method, the case of interaction situation route with two segments $\left(n_{1}=n_{2}=2\right)$ should be proved firstly. Suppose that there are two situation interaction routes:

$$
\begin{aligned}
& r_{j \rightarrow m}^{1}=\left\{\left(p_{i}, p_{i+1}\right) \mid j \longrightarrow l_{1} \longrightarrow m, i \in\{1,2\}\right\} \\
& r_{j \rightarrow m}^{2}=\left\{\left(p_{i}, p_{i+1}\right) \mid j \longrightarrow l_{2} \longrightarrow m, i \in\{1,2\}\right\}
\end{aligned}
$$

For the 1st situation interaction route, each interaction segment with formula (3):

$$
\begin{gathered}
v_{j l_{1}}=C_{j l_{1}} \cdot \frac{k_{j}+k_{l_{1}}}{2}=\left(1-\beta_{j l_{1}}\right) \cdot \frac{k_{j}+k_{l_{1}}}{2}, \\
v_{l_{1} m}=C_{l_{1} m} \cdot \frac{k_{l_{1}}+k_{m}}{2}=\left(1-\beta_{l_{1} m}\right) \cdot \frac{k_{l_{1}}+k_{m}}{2}, \\
t_{j m}^{1}=\frac{x_{j}^{*}-x_{l_{1}}}{v_{j l_{1}}}+\frac{x_{l_{1}}^{*}-x_{m}}{v_{l_{1} m}} \\
=2\left\{\left[\left(x_{j}^{*}-x_{l_{1}}\right)\left(k_{l_{1}}+k_{m}\right)+\left(x_{l_{1}}^{*}-x_{m}\right)\left(k_{j}+k_{l_{1}}\right)\right]\right. \\
\quad-\left[\beta_{l_{1} m} \cdot\left(k_{l_{1}}+k_{m}\right)\left(x_{j}^{*}-x_{l_{1}}\right)\right. \\
\left.\quad+\beta_{j l_{1}} \cdot\left(k_{j}+k_{l_{1}}\right)\left(x_{l_{1}}^{*}-x_{m}\right)\right] \\
\times\left(\left(k_{j}+k_{l_{1}}\right) \cdot\left(k_{l_{1}}+k_{m}\right)\right. \\
\quad-\left(\beta_{j l_{1}}+\beta_{l_{1} m}-\beta_{j l_{1}} \beta_{l_{1} m}\right) \\
\left.\left.\quad \cdot\left(k_{j}+k_{l_{1}}\right) \cdot\left(k_{l_{1}}+k_{m}\right)\right)^{-1}\right\} .
\end{gathered}
$$

In situation cognition stage, due to interaction position of entity $l_{1}$ and entity $l_{2}$ is in the same level, and then $x_{l_{1}} \approx$ $x_{l_{2}}$. With the target entity in situation interaction process as $x_{j}^{*}=x_{l_{1}}^{*}=1,\left(x_{j}^{*}-x_{l_{1}}\right)\left(k_{l_{1}}+k_{m}\right)$ and $\left(x_{l_{1}}^{*}-x_{m}\right)\left(k_{j}+k_{l_{1}}\right)$ are both fixed value. As $\left(x_{j}^{*}-x_{l_{1}}\right) \in[0,1]$ and $\left(k_{l_{1}}+k_{m}\right) \in[0,2]$, then $\left(k_{l_{1}}+k_{m}\right)\left(x_{j}^{*}-x_{l_{1}}\right) \in[0,2],\left(k_{j}+k_{l_{1}}\right) \cdot\left(k_{l_{1}}+k_{m}\right) \in$ $[0,4]$, and $\left(k_{l_{1}}+k_{m}\right)\left(x_{j}^{*}-x_{l_{1}}\right) \subset\left(k_{j}+k_{l_{1}}\right) \cdot\left(k_{l_{1}}+k_{m}\right)$. And $\left(\beta_{j l_{1}}+\beta_{l_{1} m}-\beta_{j l_{1}} \beta_{l_{1} m}\right)>\beta_{l_{1} m}$, so the factor as below plays decisive role in denominator:

$$
\Delta_{r \mid j \rightarrow m}^{1}=\left(\beta_{j l_{1}}+\beta_{l_{1} m}-\beta_{j l_{1}} \beta_{l_{1} m}\right) \cdot\left(k_{j}+k_{l_{1}}\right) \cdot\left(k_{l_{1}}+k_{m}\right),
$$

which is regarded as the environmental adaptability operator, and $\beta \rightarrow 0$, and then

$$
\begin{aligned}
\left.t_{j m}^{1}\right|_{\beta \rightarrow 0}=2[ & \left(\left(x_{j}^{*}-x_{l_{1}}\right)\left(k_{l_{1}}+k_{m}\right)\right. \\
& \left.+\left(x_{l_{1}}^{*}-x_{m}\right)\left(k_{j}+k_{l_{1}}\right)\right) \\
& \left.\times\left(\left(k_{j}+k_{l_{1}}\right) \cdot\left(k_{l_{1}}+k_{m}\right)\right)^{-1}\right] .
\end{aligned}
$$


With the same method, for the 2nd situation interaction route, the $\mathrm{EAO}$ is

$$
\Delta_{r \mid j \rightarrow m}^{2}=\left(\beta_{j l_{2}}+\beta_{l_{2} m}-\beta_{j l_{2}} \beta_{l_{2} m}\right) \cdot\left(k_{j}+k_{l_{2}}\right) \cdot\left(k_{l_{2}}+k_{m}\right) .
$$

Due to $\beta, k \in[0,1]$, we obtain that $\Delta_{r}^{1}, \Delta_{r}^{2}>0$. If $\Delta_{r}^{1}<$ $\Delta_{r}^{2}$, the 1st situation interaction route is chosen; otherwise, we should choose the 2 nd situation interaction route.

When there are several situation interaction routes $\left(n_{1} \geq\right.$ $2, n_{2} \geq 2 \ldots, n_{s} \geq 2$ ), the EAO of each situation interaction route is $\Delta_{r \mid j \rightarrow m}^{s_{h}}=\left(\sum_{i \in n_{j}} \beta_{i, i+1}-\prod_{i \in n_{j}} \beta_{i, i+1}\right) \cdot \prod_{i \in n_{j}}\left(k_{i}+k_{i+1}\right)$, $s_{h} \in\{1,2, \ldots, s\}$.

The best situation interaction route should be chosen with the EAO with minimum value:

$$
\begin{aligned}
\Delta_{r \mid j \rightarrow m}^{s^{*}}=\min \Delta_{r \mid j \rightarrow m}^{s_{h}} & \\
=\min [ & \left(\sum_{i \in n_{j}} \beta_{i, i+1}-\prod_{i \in n_{j}} \beta_{i, i+1}\right) \\
& \left.\cdot \prod_{i \in n_{j}}\left(k_{i}+k_{i+1}\right)\right] .
\end{aligned}
$$

Inference 1. Suppose that there are situation interaction routes in two types: merely-single interaction segment $\left(n_{1}=1\right)$ and multiple interaction segments $\left(n_{2} \geq 2\right)$, and we should take the merely-single interaction segment as the situation interaction route.

Proof. Suppose that the situation interaction route with merely-single interaction segment $\left(n_{1}=1\right)$ is $r_{j \rightarrow m}^{1}=$ $\left\{\left(p_{1}, p_{2}\right) \mid j \rightarrow m\right\}$, and then $\Delta_{r \mid j \rightarrow m}^{1}=\left(\beta_{1,2}-0\right) \cdot\left(k_{1}+k_{2}\right)=$ $\beta_{1,2} \cdot\left(k_{1}+k_{2}\right)$.

And situation interaction route with multiple interaction segments:

$$
\begin{gathered}
r_{j \rightarrow m}^{2}=\left\{\left(p_{i}, p_{i+1}\right) \mid j \longrightarrow l_{2}^{1} \longrightarrow \cdots \longrightarrow l_{2}^{q} \longrightarrow m\right\}, \\
\Delta_{r \mid j \rightarrow m}^{2}=\left(\sum_{i \in n_{2}} \beta_{i, i+1}-\prod_{i \in n_{2}} \beta_{i, i+1}\right) \cdot \prod_{i \in n_{2}}\left(k_{i}+k_{i+1}\right) .
\end{gathered}
$$

Due to $\sum_{i \in n_{2}} \beta_{i, i+1}-\prod_{i \in n_{2}} \beta_{i, i+1}>\beta_{1,2}, \prod_{i \in n_{2}}\left(k_{i}+k_{i+1}\right)>$ $\left(k_{1}+k_{2}\right)$, and then $\Delta_{r \mid j \rightarrow m}^{1}<\Delta_{r \mid j \rightarrow m}^{2}$. According to Property 1, we can obtain that $\Delta_{r \mid j \rightarrow m}^{s^{*}}=\min \left(\Delta_{r \mid j \rightarrow m}^{1}\right.$, $\left.\Delta_{r \mid j \rightarrow m}^{2}\right)=\Delta_{r \mid j \rightarrow m}^{1}$. So it is appropriate to take the merely-single interaction segment as the situation interaction route.

Property 2 (multitarget entities adjustability in situation interaction routes). Suppose that there are several situation interaction routes existing from $s$ target entities to entity $m$, and it is described as $r_{J \rightarrow m}^{s}=\left\{\left(p_{i}, p_{i+1}\right) \mid p_{i} \rightarrow p_{i+1}\right.$; $\left.p_{i}, p_{i+1} \in G_{N} \wedge\left(p_{i}^{q} \in J=\left\{j_{1}, \ldots, j_{s}\right\}, p_{i+1}^{z}=m\right)\right\}$. As difference in environment influence, environmental adaptability operator (EAO) is designed below to adjust, and we should choose the best situation interaction route with the minimum value of EAO:

$$
\Delta_{r \mid j^{*} \rightarrow m}^{s^{*}}=\min \Delta_{r \mid j_{h} \rightarrow m}^{s_{h}}\left(s_{h} \in\{1,2, \ldots, s\}\right) .
$$

Then $r_{s^{*}}$ is chosen as situation interaction route, which reflects that plan-based negotiation is from the target entity $j_{h}$ to comprise entity $m$, and other target entities have no effect on the negotiation. So multitarget entities can be adjustable in situation interaction routes.

Proof. According to recurrence method, the case of interaction situation route with two target entities $(s=2)$ should be proved firstly. Suppose that situation interaction routes with two interaction segments $\left(n_{1}=n_{2}=2\right)$ are given by

(1) $r_{j_{1} \rightarrow m}^{1}=\left\{\left(p_{i}, p_{i+1}\right) \mid j_{1} \longrightarrow l_{1} \longrightarrow m, i \in\{1,2\}\right\}$,

(2) $r_{j_{2} \rightarrow m}^{2}=\left\{\left(p_{i}, p_{i+1}\right) \mid j_{2} \longrightarrow l_{2} \longrightarrow m, i \in\{1,2\}\right\}$.

And each EAO is

$$
\begin{aligned}
& \Delta_{r \mid j_{1} \rightarrow m}^{1}=\left(\beta_{j_{1} l_{1}}+\beta_{l_{1} m}-\beta_{j_{1} l_{1}} \beta_{l_{1} m}\right)\left(k_{j_{1}}+k_{l_{1}}\right)\left(k_{l_{1}}+k_{m}\right), \\
& \Delta_{r \mid j_{2} \rightarrow m}^{2}=\left(\beta_{j_{2} l_{2}}+\beta_{l_{2} m}-\beta_{j_{2} l_{2}} \beta_{l_{2} m}\right)\left(k_{j_{2}}+k_{l_{2}}\right)\left(k_{l_{2}}+k_{m}\right) .
\end{aligned}
$$

When $\Delta_{r \mid j_{1} \rightarrow m}^{1}<\Delta_{r \mid j_{2} \rightarrow m}^{2}$, we should choose the 1st situation interaction route from target entity $j_{1}$ to compromise entity $m$, and target entity $j_{2}$ is free to search other compromise entities, which reflects the cooperation among the different target entities to improve situation interaction in high efficiency.

Similarly, for the multitarget entities $(s>2)$ and each route with multiinteraction segments $\left(n_{1}>2, \ldots, n_{s}>2\right)$, we can obtain that

$$
\begin{aligned}
\Delta_{r \mid j^{*} \rightarrow m}^{s^{*}}=\min \Delta_{r \mid j_{h} \rightarrow m}^{s_{h}} & \\
=\min & {\left[\left(\sum_{i \in n_{j}} \beta_{i, i+1}-\prod_{i \in n_{j}} \beta_{i, i+1}\right)\right.} \\
& \left.\cdot \prod_{i \in n_{j}}\left(k_{i}+k_{i+1}\right)\right] .
\end{aligned}
$$

Then we can choose the target entity $j^{*}$ in optimization to interact with compromise entity $m$, and other target entities $J / j^{*}$ are used to negotiate with other compromise entities, which are using resources efficiently to realize ODMS.

Inference 2. Suppose that there are situation interaction routes from $s$ target entities to compromise entity $m$ with two types: merely-single interaction segment $\left(n_{1}=1\right)$ and multiple interaction segments $\left(n_{2} \geq 2\right)$, and we also should take the merely-single interaction segment as the situation interaction route. 
Proof. Suppose that situation interaction route with merelysingle interaction segment $\left(n_{1}=1\right)$ is $r_{j_{1} \rightarrow m}^{1}=\left\{\left(p_{1}, p_{2}\right) \mid\right.$ $\left.j_{1} \rightarrow m\right\}$, and then $\Delta_{r \mid j_{1} \rightarrow m}^{1}=\left(\beta_{1,2}-0\right) \cdot\left(k_{1}+k_{2}\right)=\beta_{1,2}$. $\left(k_{1}+k_{2}\right)$.

From target entities $j_{k}(k \in\{2,3, \ldots, s\})$ to entity $\mathrm{m}$, situation interaction routes with several interaction segments $\left(n_{k} \geq 2\right)$ are given by $r_{j_{k} \rightarrow m}^{k}=\left\{\left(p_{i}, p_{i+1}\right) \mid j_{k} \rightarrow l_{k 2}^{1} \rightarrow\right.$ $\left.\cdots \rightarrow l_{k_{-} 2}^{q} \rightarrow m\right\}$. Then we can gain that $\Delta_{r \mid j_{k} \rightarrow m}^{k}=$ $\min \left[\left(\sum_{i \in n_{k}} \beta_{i, i+1}-\prod_{i \in n_{k}} \beta_{i, i+1}\right) \cdot \sum_{i \in n_{k}}\left(k_{i}+k_{i+1}\right)\right]$.

Within the same environment influence as $\beta_{1,2}=\beta_{j_{1} m} \approx$ $\beta_{i, i+1}$ (for all $i \in n_{k}$ ), then we can obtain $\sum_{i \in n_{2}} \beta_{i, i+1}-$ $\prod_{i \in n_{2}} \beta_{i, i+1}>\beta_{1,2}$. So $\prod_{i \in n_{2}}\left(k_{i}+k_{i+1}\right)>\left(k_{1}+k_{2}\right)$, $\Delta_{r \mid j_{1} \rightarrow m}^{1}<\Delta_{r \mid j_{k} \rightarrow m}^{k}$. According to Property 2, we can deduce that $\Delta_{r \mid j^{*} \rightarrow m}^{s^{*}}=\min \Delta_{r \mid j_{h} \rightarrow m}^{s_{h}}=\Delta_{r \mid j_{1} \rightarrow m}^{1}$. And the situation interaction route with merely-single interaction segment is suitable to be adopted.

In order to adapt the influence of environment change, combining Properties 1 and 2, strategies below should be taken to find the situation interaction route quickly, which can improve ODMS capability.

(1) In the case target entity and compromise entity are both fixed, according to Property 1 and Inference 1 , and when $\beta$ is low, we should choose $k_{j}$, namely, EID, with high value in the situation interaction route, which can reduce the interaction limit time $\left.t_{j m}^{s^{*}}\right|_{\beta \rightarrow 0}$. Otherwise, when $\beta$ is in high value, choosing the entity with $k_{j}$ in high value will cause communication blocked severely, so we should choose entity with $k_{j}$ in low value, and then $\Delta_{r \mid j \rightarrow m}^{s^{*}}$ is also reduced, which can make $\left.t_{j m}^{s^{*}}\right|_{\beta>0}$ depress.

(2) In the case about target entities are uncertainty but compromise entities are fixed, according to Property 2 and Inference 2, we should choose situation interaction route with merely-single interaction segment in possible, which can be used to confirm the suitable target entities and form the situation interaction route in the whole $\mathrm{C} 2$ organization.

Thus ODMS strategies set of choosing situation interaction routes under environment influence is:

$$
R=\left\{r_{\mathrm{st}_{i} \rightarrow \mathrm{en}_{i}}^{s_{i}^{*}} \mid \mathrm{st}_{i} \vee \mathrm{en}_{i} \in G_{N}, i \in N\right\}
$$

where $s_{i}^{*}$ is the situation interaction route in optimization within $\mathrm{st}_{i} \rightarrow \mathrm{en}_{i}$.

4.2. ODMS Strategies within Time Restriction. In the actual operation, in order to ensure the cooperation action among operational entities, ODMS is usually incomplete within the time restriction, and there are certain accepted synchronization errors in ODMS. We can summarize properties as follows.
Property 3 (error accumulation in incomplete ODMS). Due to that $\gamma \leq u_{i} \leq 1$ and the SIQ of each situation interaction segment is in little difference, so $u\left(t_{k}\right) \approx u\left(t_{l}\right) \cdots u\left(t_{m}\right)$, which are marked as $u$ in unified. Accepted synchronization error in the interaction segment $m \rightarrow j$, in which entity $j$ is taken as target entity, is approximate described as

$$
\begin{aligned}
\Delta x_{j m} \approx & \left(1-u^{n}\right) \cdot\left(x_{j}^{*}-x_{k}\left(t_{k}^{\prime}\right)\right) \\
\approx & \left(1-u\left(t_{k}\right) \cdot u\left(t_{l}\right) \cdots u\left(t_{m}\right)\right) \\
& \cdot\left(x_{j}^{*}-x_{k}\left(t_{k}^{\prime}\right)\right),
\end{aligned}
$$

where $u\left(t_{k}\right)$ is the SIQ of entity $k$.

Proof. According to recurrence method, the process of the interaction segment of $j \rightarrow k$ can be described as $x_{k}^{*}=$ $x_{k}\left(t_{k}^{\prime}\right)-u\left(t_{k}\right) \cdot\left[x_{k}\left(t_{k}^{\prime}\right)-x_{j}^{*}\right]$. Then the local synchronization error is

$$
\Delta x_{j k}=x_{j}^{*}-x_{k}^{*}=\left(1-u\left(t_{k}\right)\right) \cdot\left(x_{j}^{*}-x_{k}\left(t_{k}^{\prime}\right)\right) .
$$

In the situation interaction segment $k \rightarrow l$, we can obtain that

$$
\begin{aligned}
x_{l}^{*}= & x_{l}\left(t_{l}^{\prime}\right)-u\left(t_{l}\right) \cdot\left[x_{l}\left(t_{l}^{\prime}\right)-x_{k}^{*}\right] \\
= & \left(1-u\left(t_{l}\right)\right) x_{l}\left(t_{l}^{\prime}\right) \\
& +u\left(t_{l}\right)\left(1-u\left(t_{k}\right)\right) \cdot x_{k}\left(t_{k}^{\prime}\right)+u\left(t_{l}\right) u\left(t_{k}\right) \cdot x_{j}^{*} .
\end{aligned}
$$

As $u\left(t_{l}\right) \approx u\left(t_{k}\right)$ and both of them are marked as $u$, then

$$
\begin{aligned}
\Delta x_{j l}= & x_{j}^{*}-x_{l}^{*} \approx(1-u) \cdot\left(x_{j}^{*}-x_{l}\left(t_{l}^{\prime}\right)\right) \\
& +u(1-u) \cdot\left(x_{j}^{*}-x_{k}\left(t_{k}^{\prime}\right)\right) .
\end{aligned}
$$

Due to $x_{l}\left(t_{l}^{\prime}\right) \approx x_{k}\left(t_{k}^{\prime}\right)$, we can gain that $\Delta x_{j l}=\left(1-u^{2}\right)$. $\left(x_{j}^{*}-x_{k}\left(t_{k}^{\prime}\right)\right) \approx\left(1-u\left(t_{k}\right) u\left(t_{l}\right)\right) \cdot\left(x_{j}^{*}-x_{k}\left(t_{k}^{\prime}\right)\right)$. If $u=1$, then $\Delta x_{j l}=0$. Else if $0<u<1$, we can obtain that $0<$ $\Delta x_{j k}<\Delta x_{j l}$, and synchronization accumulation is enlarged through continuous situation interaction. So according to analogy analysis in the situation interaction route, we can gain Property 3.

Property 4 (time adjustability in incomplete ODMS). In incomplete ODMS, time adjustability of negotiation with concession pattern is the best of the three negotiation patterns. The time consumed in concession pattern is less than other two negotiation patterns with the same SIQ, which provides larger time range for subsequent interaction segments to adjust and fulfill the time restriction.

Proof. In the situation interaction segment $j \rightarrow k$, time consumed of complete ODMS with the $i$ th negotiation pattern is $t_{i}^{*}$, and time consumed of incomplete ODMS $(u=$ $b<1)$ is $t_{i}$ correspondingly, then $t_{i}<t_{i}^{*}(i \in\{1,2,3\})$, and $\Delta t_{1}=t_{1}^{*}-t_{1}=(a-b) / v_{k l}^{\prime}, \Delta t_{2}=t_{2}^{*}-t_{2}=(\sqrt{a}-\sqrt{b}) / v_{k l}^{\prime}, \Delta t_{3}=$ $t_{3}^{*}-t_{3}=\left(a^{2}-b^{2}\right) / v_{k l}^{\prime}$. 


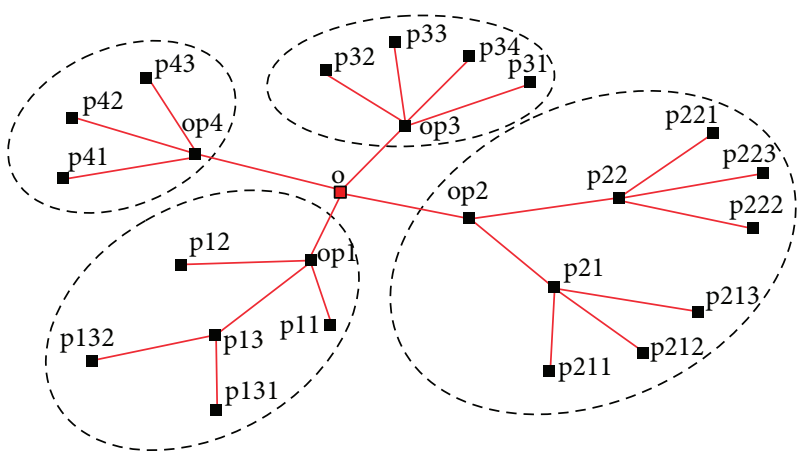

(a) Target entity o

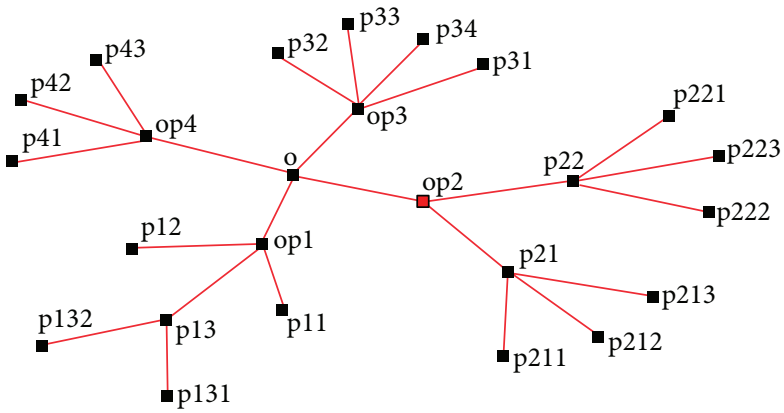

(b) Target entity op2

FIGURE 4: C2 organization with tree structure: NET1.

Due to $a=1$ and $0<b<1$, then $a+b>1, \sqrt{a}+\sqrt{b}>1$, so $\sqrt{a}-\sqrt{b}<a-b<a^{2}-b^{2}$. Then we can deduce that $\Delta t_{3}>\Delta t_{1}>\Delta t_{2}$. As known in Figure 3 , the time $t_{3}$ consumed in concession negotiation process is much less than $t_{3}^{*}$ in the incomplete ODMS with $u=0.95$, which reflects the best time adjustability in three negotiation patterns. With the decrease of $u$, the difference of gap is enlarged in the different negotiation process.

Combined Properties 3 and 4, when the performance of ODMS cannot fulfill the time restriction, we should take following strategies to adjust the performance of ODMS.

(1) Adjusting time consumed in situation interaction segment with low EID operational entities in any possible way: according to Property 3 , synchronization error of operation entities with high EID will cause error accumulation in following situation interaction.

(2) Adopting the concession pattern in negotiation in any possible way: according to Property 4 , when target entity is changed, structure of $\mathrm{C} 2$ organization should be adjusted for controlling more resource quickly, which can transfer the target entity to be the most important entity for concession negotiation.

Based on the analysis above, ODMS strategies set within time restriction can be constructed as $U=\left\{u_{i} \mid \gamma \leq u_{i} \leq\right.$ $1, i \in N\}$, where $u_{i}$ is the SIQ of each interaction segment.

\section{Simulation Cases}

5.1. Parameters Setting. Taking the operational organization in [25], for example, the organization is abstracted as network of $\mathrm{C} 2$ organization with 25 nodes and 4 command corporations shown in Figure 4, whose average degree is $\langle k\rangle=1.92$, and parameters are initialized as seen in Table 4. Suppose that SPL is uniform distribution within $[0,0.1]$, and entity o with the highest EID is induced as target entity with facility.

\subsection{ODMS Performance in Continuous Changing Situation.} In the ideal condition $(\beta=0)$, according to parameters setting above, stable situation (as special continuous changing situation) and three continuous changing situation patterns
TABLE 4: Initialization parameters of $\mathrm{C} 2$ organization.

\begin{tabular}{|c|c|c|c|c|c|}
\hline \multirow{2}{*}{ No. } & \multirow{2}{*}{ ID } & \multirow{2}{*}{$x_{i 0}$} & NET1 & NET2 & NET3 \\
\hline & & & $\mathrm{NID}_{1}$ & $\mathrm{NID}_{2}$ & $\mathrm{NID}_{3}$ \\
\hline 1 & o & 0.0203 & 0.7100 & 0.5300 & 0.2866 \\
\hline 2 & op1 & 0.0199 & 0.5242 & 0.4426 & 0.4150 \\
\hline 3 & op2 & 0.0604 & 0.6608 & 0.6892 & 0.6550 \\
\hline 4 & op3 & 0.0272 & 0.4742 & 0.3792 & 0.4644 \\
\hline 5 & op4 & 0.0199 & 0.4042 & 0.4152 & 0.3916 \\
\hline 6 & p11 & 0.0015 & 0.0850 & 0.0700 & 0.0634 \\
\hline 7 & p12 & 0.0747 & 0.0850 & 0.0700 & 0.0634 \\
\hline 8 & p13 & 0.0445 & 0.2416 & 0.2266 & 0.2200 \\
\hline 9 & p131 & 0.0932 & 0.0500 & 0.0500 & 0.0500 \\
\hline 10 & p132 & 0.0466 & 0.0500 & 0.0500 & 0.0500 \\
\hline 11 & $\mathrm{p} 21$ & 0.0419 & 0.3900 & 0.3360 & 0.3326 \\
\hline 12 & p211 & 0.0846 & 0.0550 & 0.0550 & 0.0550 \\
\hline 13 & p212 & 0.0525 & 0.0550 & 0.0550 & 0.0550 \\
\hline 14 & p213 & 0.0203 & 0.0550 & 0.0550 & 0.0550 \\
\hline 15 & p22 & 0.0672 & 0.3900 & 0.3360 & 0.3326 \\
\hline 16 & p221 & 0.0838 & 0.0550 & 0.0550 & 0.0550 \\
\hline 17 & p222 & 0.0020 & 0.0550 & 0.0550 & 0.0550 \\
\hline 18 & p223 & 0.0681 & 0.0550 & 0.0550 & 0.0550 \\
\hline 19 & p31 & 0.0379 & 0.0574 & 0.0574 & 0.0466 \\
\hline 20 & p32 & 0.0832 & 0.0574 & 0.0574 & 0.0466 \\
\hline 21 & p33 & 0.0503 & 0.0574 & 0.0574 & 0.0466 \\
\hline 22 & p34 & 0.0709 & 0.0574 & 0.0574 & 0.0466 \\
\hline 23 & $\mathrm{p} 41$ & 0.0429 & 0.0550 & 0.0440 & 0.0422 \\
\hline 24 & $\mathrm{p} 42$ & 0.0305 & 0.0550 & 0.0440 & 0.0422 \\
\hline 25 & $\mathrm{p} 43$ & 0.0190 & 0.0550 & 0.0440 & 0.0422 \\
\hline
\end{tabular}

are given together in Table 5. As limit of paper, the sudden changing situation within $t \in[0,5]$ and the IPA of each entity are analyzed in Figure 5.

The axis of abscissa shows time consumed and axis of ordinate express SCL, and then complete ODMS under four changing situations, shown in Figure 6, can be described as curve cluster about the SCL changing process of 25 operational entities. 


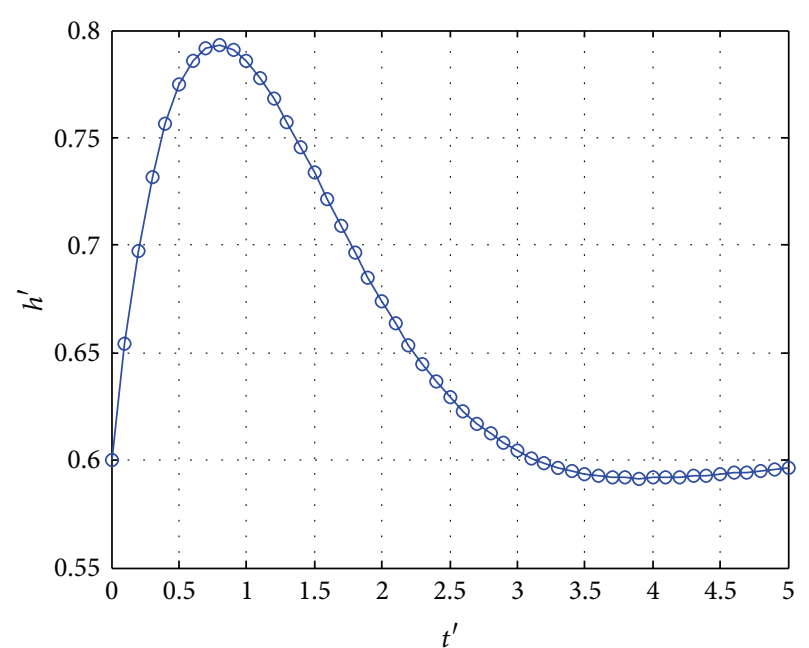

(a) Situation changing process

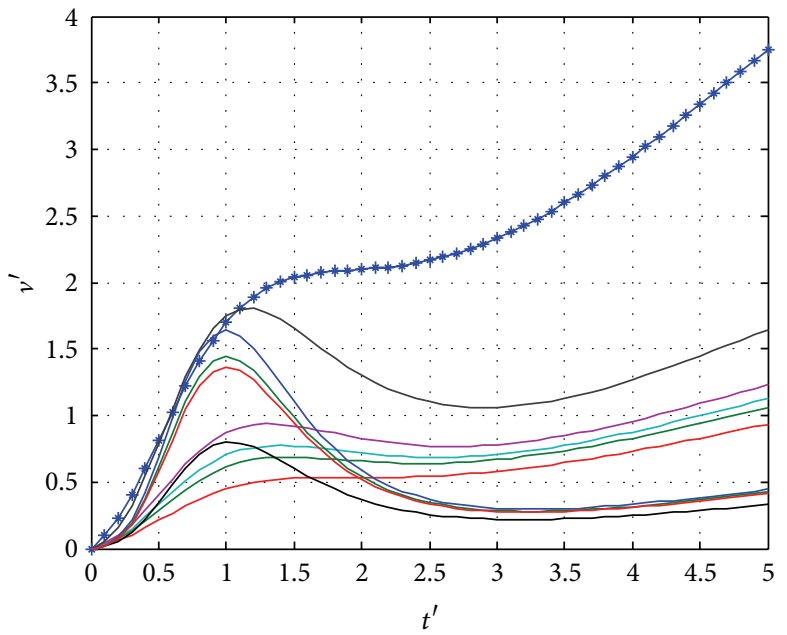

(b) IPA of operational entities

FIGURE 5: Situation in sudden changing pattern.

TABLE 5: The SCR in continuous changing situation.

\begin{tabular}{lll}
\hline No. & Situation change patterns & SCR \\
\hline 1 & Stable situation & $h_{t}^{\prime}=0$ \\
2 & Identical changing situation & $h_{t}^{\prime}=0.8$ \\
3 & Gradual changing situation & $h_{t}^{\prime}=0.17 * t+0.2$ \\
4 & Sudden changing situation & $h_{t}^{\prime}=0.6 *\left(1-\sin (t-\pi) * e^{-t}\right)$ \\
\hline
\end{tabular}

With two phases in ODMS process, STE can be obtained on the basis of time consuming within two stages correspondingly and shown in Figure 7(a). Meanwhile, SQ can be gained with cognition change in two stages and shown in Figure 7(b). Other measure indexes of ODMS are listed in Table 6. From the statistics in Table 6, with the increase of SCR, the ST is shortened correspondingly.

As in process P1 in Figure 6(a), due to little change in the situation, there are fewer nodes updating situation information. Then long time is consumed in situation cognition stage, but the SCL is not improved highly, which leads to more time used to negotiate in situation interaction stage, and ST is extended in the ODMS, as P1 process in Figure 7.

As in process P2 and process P3 in Figures 6(b) and 6(c) respectively, with SCR increasing, the number of nodes updating information raises correspondingly, and then IPA and SCL in situation cognition stage are improved in a short time. Compared with process $\mathrm{P} 1$, time consumed in situation interaction stage is shortened, and then ST can be reduced, which is shown as process $\mathrm{P} 2$ and process P3 in Figure 7.

As process P4 in Figure 6(d), the SCL of each operational entity improves with SCR increasing. When situation suddenly has a great change, it leads to situation information explosion apperceived by $\mathrm{C} 2$ organization in a short time. The communication routes are easy to be blocked, which can make the IPA decrease among the operational nodes and the SCL reduced (as subgraph shown in Figure 10 within time interval $[1,2])$. After the decrease of SCR, the C2
TABLE 6: Indexes list of ODMS.

\begin{tabular}{lccccc}
\hline No. & ID & $T$ & $\gamma_{i o}$ & $\tau$ & $\chi$ \\
\hline 1 & P1 & 24.3425 & 1 & 1.4420 & 6.7622 \\
2 & P2 & 15.2562 & 1 & 1.8034 & 2.1064 \\
3 & P3 & 14.2024 & 1 & 1.8306 & 1.5813 \\
4 & P4 & 11.9370 & 1 & 1.7543 & 1.2449 \\
\hline
\end{tabular}

organization's ability of efficiently processing and interpreting information recovers gradually, and SCL of each entity is still improved. Due to overload processing of situation information, the operational nodes have the highest SCL while consuming the minimum time, which is benefit for realizing ODMS in shortest time, shown in Figure 7.

According to above analysis, under continuous changing situation, the situation cognition stage is the foundation of situation interaction stage, which directly influences the STE and SQ of ODMS. When the target entity is transferred from o to op2, the ODMS performance of $\mathrm{C} 2$ organization with tree structure drops obviously under the stable situation (shown in Figure 8), which denotes that it is weak for C2 organization with tree structure to adapt situation change.

So in order to adapt the complex environment change, C2 organization should make entities obtain IPA as many as possible. The main ways are changing organization structure and selecting appropriate strategy to complete the situation interaction process. The following sections about two aspects strategies are used to improve the ODMS performance within environment influence and time restriction separately.

5.3. ODMS Strategies under Environment Influence. Taken stable situation as an example, environment influence factor $\beta=[0,0.2]$ is introduced to analyze adaptive ability of C2 organization with different structures. Then the crossstructure $(\langle k\rangle=2.02)$ is constructed on the basis of tree structure shown in Figure 9. 


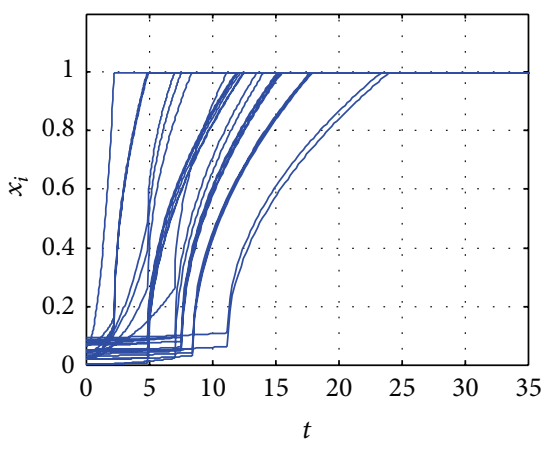

(a) Stable situation: P1

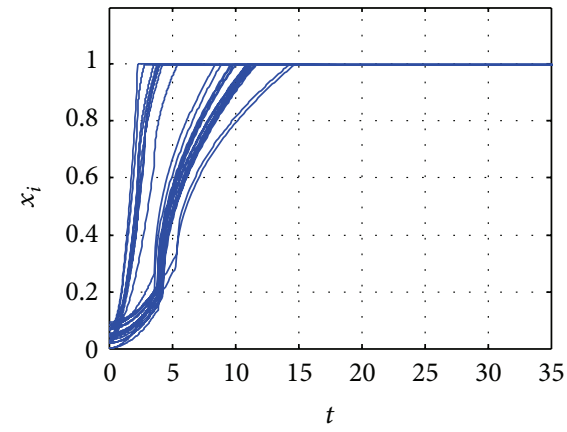

(b) Identical changing situation: P2

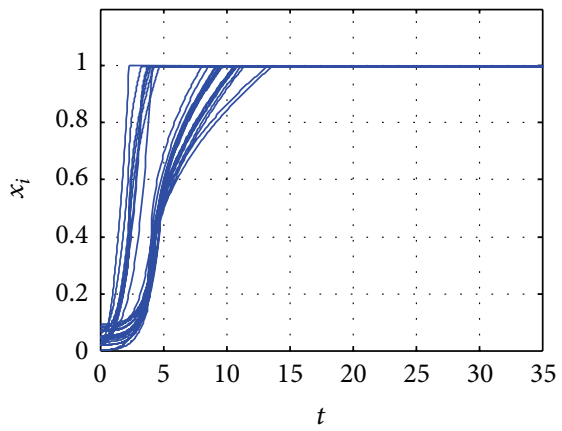

(c) Gradual changing situation: P3
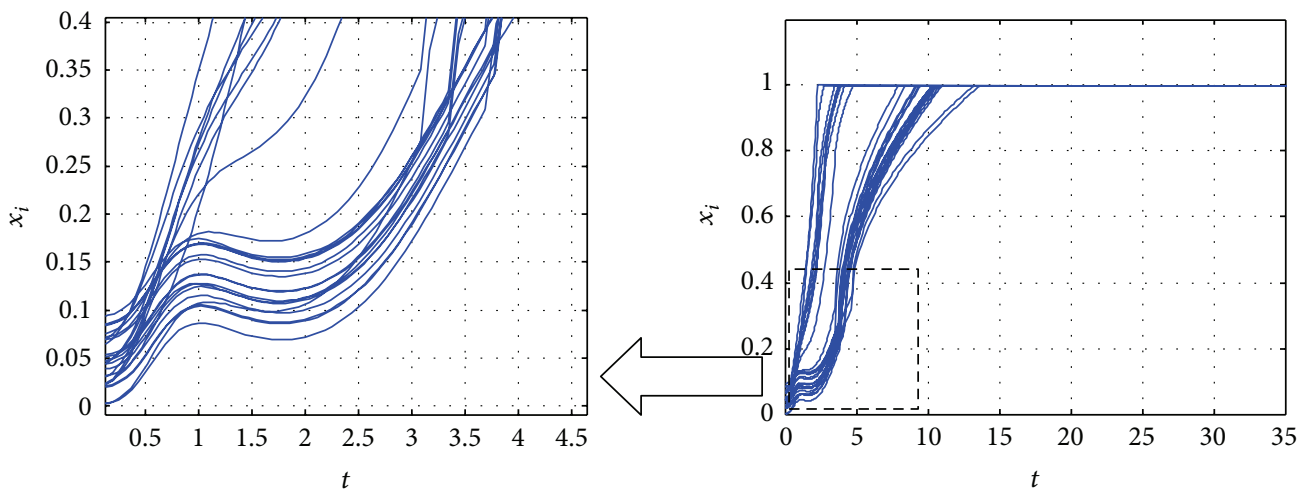

(d) Sudden changing situation: P4

FIgURE 6: Complete ODMS under four changing situation.

When $\beta=0$, the situation interaction route in NET1 is still adopted in NET2, and the ODMS performance is shown in Figure 15. When $\beta=[0,0.2]$, it can reduce the communication unblocked degree $C$. Taking op 2 as target entity, ODMS strategies set of choosing situation interaction route is analyzed in Table 7 according to Properties 1 and 2, and $\left({ }^{*}\right)$ denotes the chosen situation interaction routes.

In Table $7, r_{\mathrm{op} 2 \rightarrow \mathrm{p} 31}^{1^{*}}, r_{\mathrm{op} 2 \rightarrow \mathrm{p} 41}^{1^{*}}$, and $r_{\mathrm{op} 2 \rightarrow \mathrm{p} 12}^{2^{*}}$ are the best situation interaction route separately with Property 1. Moreover, for the target entities op 2 and $\mathrm{p} 21, r_{\mathrm{op} 2 \rightarrow \mathrm{p} 211}^{1^{*}}$ and $r_{\mathrm{op} 2 \rightarrow \mathrm{p} 221}^{2^{*}}$ are the best situation interaction route to p22, respectively, according to Property 2. So the situation interaction routes in NET2 are given as $R_{\mathrm{NET} 2}=$ $\left\{r_{\mathrm{op} 2 \rightarrow \mathrm{p} 31}^{1^{*}}, r_{\mathrm{op} 2 \rightarrow \mathrm{p} 41}^{1^{*}}, r_{\mathrm{op} 2 \rightarrow \mathrm{p} 12}^{2^{*}}, r_{\mathrm{op} 2 \rightarrow \mathrm{p} 211}^{1^{*}}, r_{\mathrm{op} 2 \rightarrow \mathrm{p} 221}^{2^{*}}\right\}$.
Comparing Figure 8 with Figure 10(a), networked level of NET2 is higher than NET1, then and IPA of NET2 improves but shortens its ST under stable situation with $\beta=0$. Comparing Figure 9 with Figure 10(b), although environment influence factor is increased $(\beta=[0,0.2])$, situation interaction routes chosen in reason can improve the quality of situation interaction, which also improves the ODMS performance. So the adaptive ability of NET2 with crossstructure is stronger than NET1 with tree structure. Based on this, C2 organization with network structure is generated by further extending networked level in Figure 11, then ODMS performance is shown in Figure 12, and strategies of choosing situation interaction route are listed in Table 8.

Contrastive analysis in both NET3 and NET2, network degree of NET3 is higher than NET2, namely, $\langle k\rangle=2.24$. It 


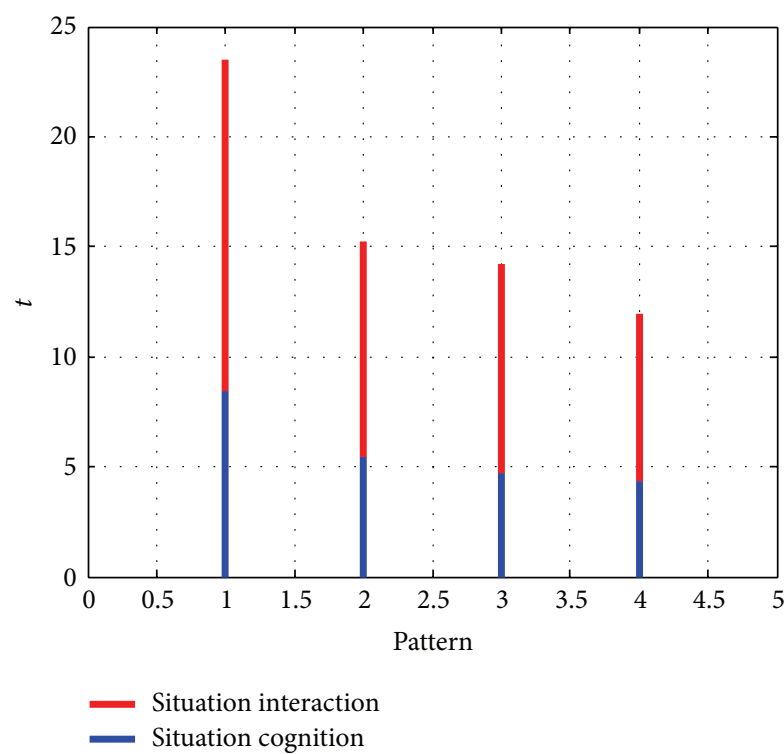

(a) The STE analysis

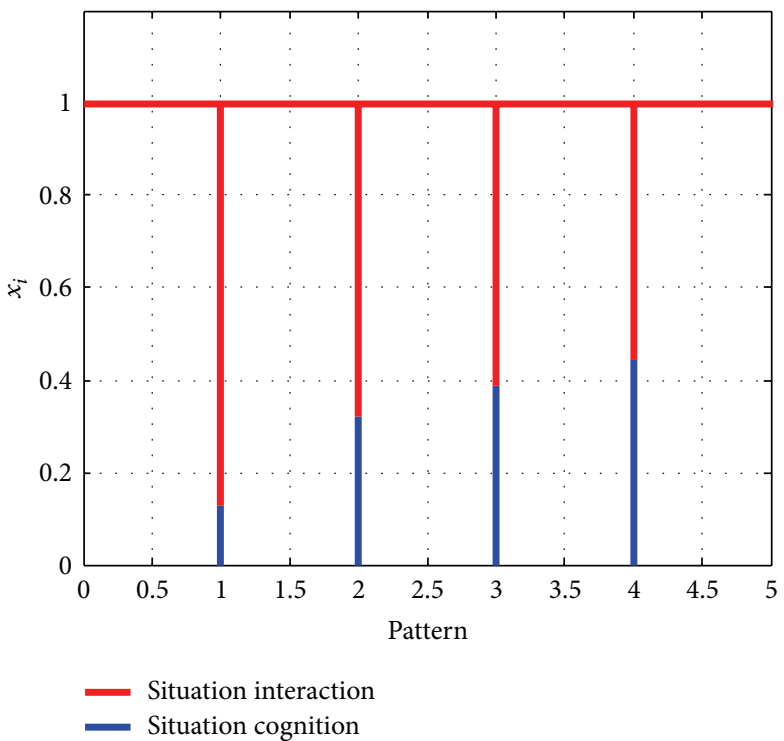

(b) The SQ analysis

FIGURE 7: The STE and SQ under different changing situations.

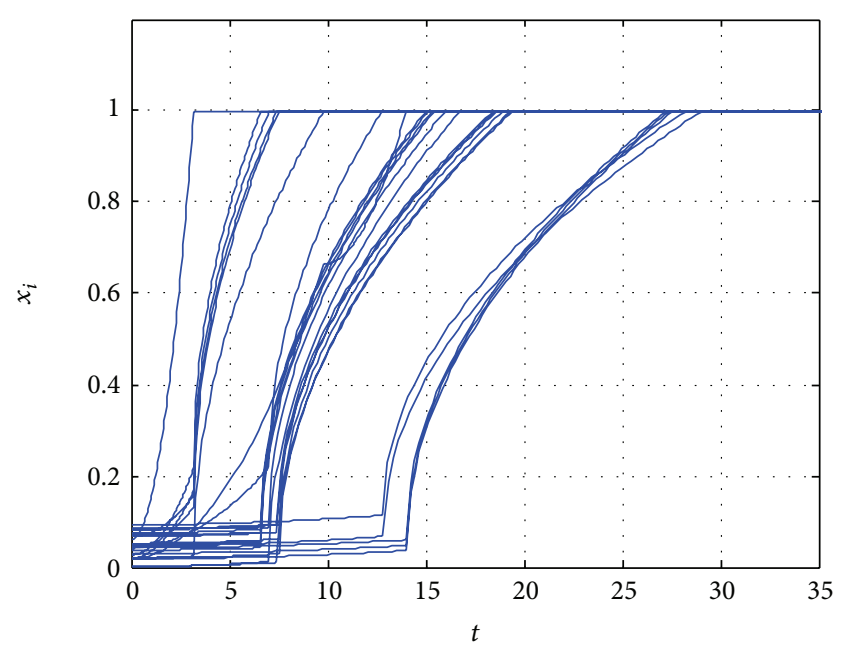

Figure 8: Complete ODMS in NET1 under stable situation with target entity op2: P5.

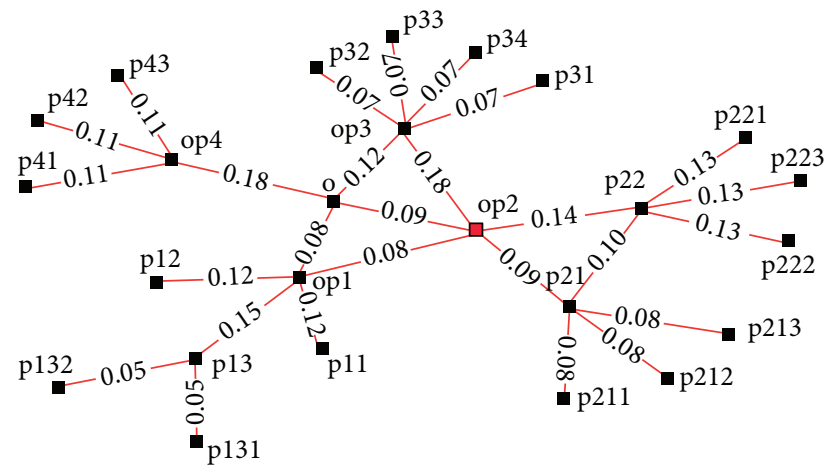

FIGURE 9: C2 organization with cross structure: NET2.
TABLE 7: Situation interaction routes within NET1.

\begin{tabular}{|c|c|c|c|}
\hline$\underline{R_{\mathrm{st}_{i} \rightarrow \mathrm{en}_{i}}}$ & No. & Situation interaction routes & $\Delta_{r}$ \\
\hline \multirow{2}{*}{$r_{\mathrm{op} 2 \rightarrow \mathrm{p} 31}$} & $1^{*}$ & op2 $\rightarrow$ op3 $\rightarrow$ p31 & 0.1107 \\
\hline & 2 & $\mathrm{op} 2 \rightarrow \mathrm{o} \rightarrow \mathrm{op} 3 \rightarrow \mathrm{p} 31$ & 0.1351 \\
\hline \multirow{3}{*}{$r_{\mathrm{op} 2} \rightarrow \mathrm{p} 41$} & $1^{*}$ & op $2 \rightarrow$ o $\rightarrow$ op $4 \rightarrow \mathrm{p} 43$ & 0.2001 \\
\hline & 2 & $\mathrm{op} 2 \rightarrow \mathrm{op} 1 \rightarrow \mathrm{o} \rightarrow \mathrm{op} 4 \rightarrow \mathrm{p} 43$ & 0.2149 \\
\hline & 3 & op $2 \rightarrow$ op $3 \rightarrow$ o $\rightarrow$ op $4 \rightarrow$ p 43 & 0.2486 \\
\hline \multirow{2}{*}{$r_{\mathrm{op} 2 \rightarrow \mathrm{p} 12}$} & 1 & $\mathrm{op} 2 \rightarrow \mathrm{o} \rightarrow \mathrm{op} 1 \rightarrow \mathrm{p} 12$ & 0.1757 \\
\hline & $2^{*}$ & op $2 \rightarrow$ op $1 \rightarrow$ p12 & 0.1105 \\
\hline \multirow[b]{2}{*}{$r_{\mathrm{op} 2} \rightarrow \mathrm{p} 211$} & $1^{*}$ & op $2 \rightarrow$ p $21 \rightarrow$ p211 & 0.0653 \\
\hline & 2 & $\mathrm{op} 2 \rightarrow \mathrm{p} 22 \rightarrow \mathrm{p} 21 \rightarrow \mathrm{p} 211$ & 0.0859 \\
\hline \multirow[b]{2}{*}{$r_{\mathrm{op} 2 \rightarrow \mathrm{p} 221}$} & 1 & op $2 \rightarrow$ p $22 \rightarrow$ p221 & 0.1009 \\
\hline & $2^{*}$ & $\mathrm{op} 2 \rightarrow \mathrm{p} 21 \rightarrow \mathrm{p} 22 \rightarrow \mathrm{p} 211$ & 0.0859 \\
\hline
\end{tabular}

${ }^{*}$ means that route with the symbol is the best situation interaction route from the fixed target entity to the fixed compromise entity and can be selected to negotiate.

has benefit to allocating decision rights, which is good for adjusting situation interaction routes to adapt environment influence. From entity op2 to entity p31, $r_{\mathrm{op} 2 \rightarrow \mathrm{p} 31}^{2^{*}}: \mathrm{op} 2 \rightarrow$ $\mathrm{o} \rightarrow \mathrm{op} 3 \rightarrow \mathrm{p} 31$ in Table 8 and $r_{\mathrm{op} 2 \rightarrow \mathrm{p} 31}^{1^{*}}: \mathrm{op} 2 \rightarrow \mathrm{op} 3 \rightarrow \mathrm{p} 31$ in Table 7 are the results of allocating decision rights agilely. So situation interaction routes in NET3 are given as $R_{\mathrm{NET} 3}=$ $\left\{r_{\mathrm{op} 2 \rightarrow \mathrm{p} 31}^{2^{*}}, r_{\mathrm{op} 2 \rightarrow \mathrm{p} 41}^{4^{*}}, r_{\mathrm{op} 2 \rightarrow \mathrm{p} 12}^{1^{*}}, r_{\mathrm{op} 2 \rightarrow \mathrm{p} 211}^{1^{*}}, r_{\mathrm{op} 2 \rightarrow \mathrm{p} 221}^{2^{*}}\right\}$.

5.4. ODMS Strategies within Time Restriction. Suppose that, in order to realize cooperation among operational entities, the time restriction is within 20 unit time (remarked as red vertical real line in Figure 12), and the accepted synchronization error within 5\%, and then SE should reach $95 \%$ at least (remarked as red horizontal dotted line in Figure 12) Then 


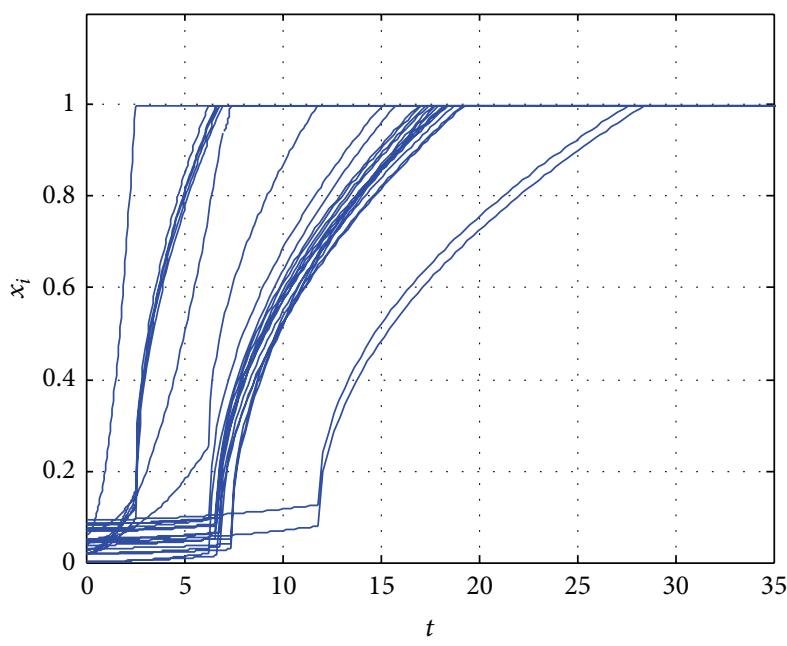

(a) $\beta=0$

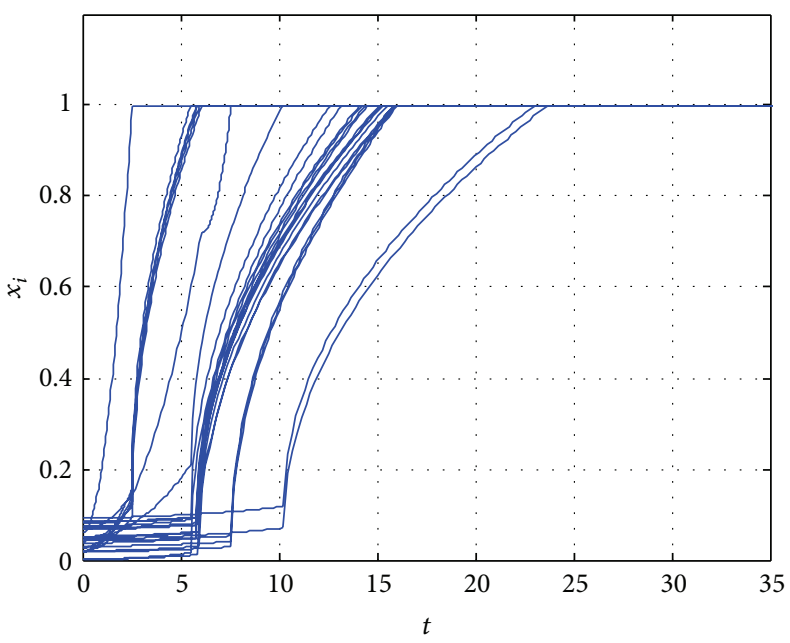

(b) $\beta=[0,0.2]$

Figure 10: Complete ODMS in NET2 with different $\beta$.

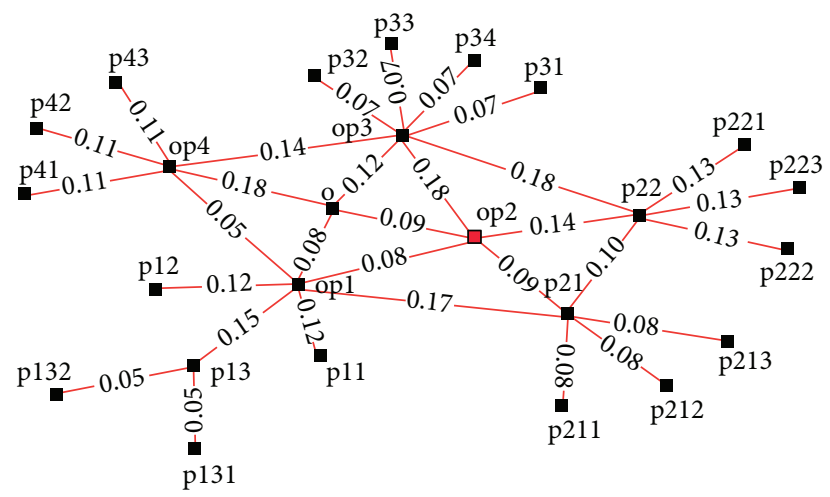

FIGURE 11: C2 organization with network structure: NET3.

ODMS performance is acceptable under other three situation changing patterns with NET1 but the stable situation with NET3. As communication is blocked in some extent within NET3, it takes long time for situation interaction, which makes ST beyond the time restriction.

As shown in sub graph of Figure 12, entities p132 and p131 do not fulfill the time restriction. The strategies set of incomplete ODMS is given as $U P_{1}^{1}$ and $U P_{1}^{2}$ in Table 9, and the processes of incomplete ODMS are shown in Figure 13.

Because that entity op 2 is the most important entity in NET3 (shown in Table 4), other entities should adopt concession negotiation pattern to realize situation interaction in high quality, so the ODMS performance is new, and the ST of C2 organization is in low adjustability. Following the strategies in Table 9, the ST of incomplete ODMS is shortened with 0.772 and 0.765 , respectively, and comprehensive synchronization extent $S$ is 0.9946 and 0.9967 , respectively, which can fulfill the operation demand.

\section{Discussion}

As known in simulation cases, ODMS with three phases is studied in detail in the paper. Then compared with models
TABLE 8: Situation interaction routes within NET2.

\begin{tabular}{|c|c|c|c|}
\hline$R_{\mathrm{st}_{i} \rightarrow \mathrm{en}_{i}}$ & No. & Situation interaction routes & $\Delta_{r}$ \\
\hline \multirow{2}{*}{$r_{\mathrm{op} 2 \rightarrow \mathrm{p} 31}$} & 1 & op $2 \rightarrow$ op $3 \rightarrow$ p 31 & 0.1255 \\
\hline & $2^{*}$ & op2 $\rightarrow$ o $\rightarrow$ op3 $\rightarrow$ p31 & 0.0583 \\
\hline \multirow{4}{*}{$r_{\mathrm{op} 2 \rightarrow \mathrm{p} 41}$} & 1 & op $2 \rightarrow$ op $1 \rightarrow$ op $4 \rightarrow$ p 41 & 0.0017 \\
\hline & 2 & op2 $\rightarrow$ o $\rightarrow$ op $4 \rightarrow$ p 41 & 0.0020 \\
\hline & 3 & $\mathrm{op} 2 \rightarrow \mathrm{op} 3 \rightarrow \mathrm{op} 4 \rightarrow \mathrm{p} 41$ & 0.0034 \\
\hline & $4^{*}$ & $\mathrm{op} 2 \rightarrow \mathrm{o} \rightarrow \mathrm{op} 1 \rightarrow \mathrm{op} 4 \rightarrow \mathrm{p} 41$ & 0.0015 \\
\hline \multirow{2}{*}{$r_{\mathrm{op} 2} \rightarrow \mathrm{p} 12$} & $1^{*}$ & op $2 \rightarrow$ o $\rightarrow$ op $1 \rightarrow$ p12 & 0.0916 \\
\hline & 2 & op $2 \rightarrow$ op1 $\rightarrow$ p12 & 0.0975 \\
\hline \multirow[b]{2}{*}{$r_{\mathrm{op} 2} \rightarrow \mathrm{p} 211$} & $1^{*}$ & op2 $\rightarrow$ p21 $\rightarrow$ p211 & 0.0623 \\
\hline & 2 & $\mathrm{op} 2 \rightarrow \mathrm{p} 22 \rightarrow \mathrm{p} 21 \rightarrow \mathrm{p} 211$ & 0.0812 \\
\hline \multirow{2}{*}{$r_{\mathrm{op} 2} \rightarrow \mathrm{p} 221$} & 1 & op $2 \rightarrow$ p $22 \rightarrow$ p 221 & 0.0964 \\
\hline & $2^{*}$ & $\mathrm{op} 2 \rightarrow \mathrm{p} 21 \rightarrow \mathrm{p} 22 \rightarrow \mathrm{p} 221$ & 0.0812 \\
\hline
\end{tabular}

${ }^{*}$ means that route with the symbol is the best situation interaction route from the fixed target entity to the fixed compromise entity and can be selected to negotiate.

referred to in Section 1, the advantages and shortcomings of the ODMS model are analyzed and discussed in this paper.

For the group communication model in $[5,6]$, it does not refer to the opinion formation. Taking the incomplete ODMS process $P_{1}^{0}$ in Figure 12 as example, the SCL in the time of $t=13$ is regarded as original opinions, and communication parameters in [5] are introduced as opinion accepted degree $u=0.2$ and communication allow degree $d=0.2$. Suppose that 1 unit time is consumed in the each communication process in the whole $\mathrm{C} 2$ organization, and then the results of two methods can be expressed in the same picture. After 25 units time, the synchronization process in [5] is shown with red real line in Figure 14. As communication processes have no effect on the SCL improvement of certain entity in the end, it can hardly get to the unanimous status, which also makes ODMS realization in hard.

For the models of CE and CSSync in [13], they do not refer to cognitive formation but only the difference of cognition. 


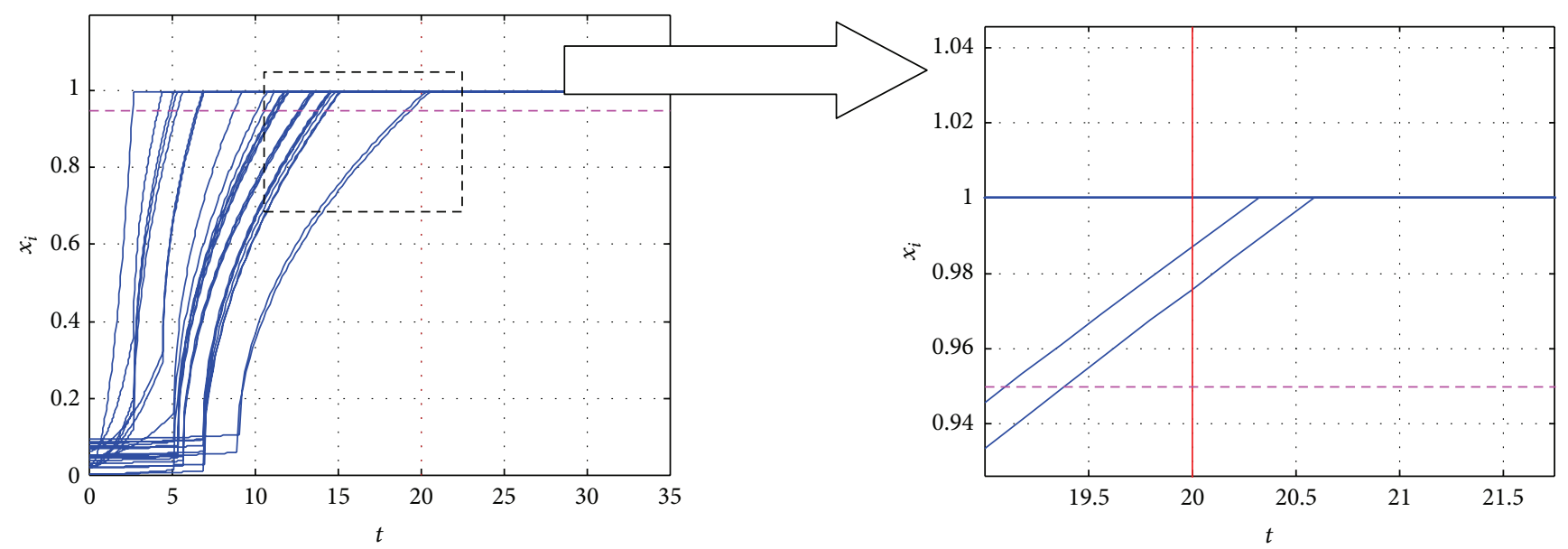

FIgure 12: Complete ODMS in NET3 with $\beta=[0,0.2]$ as $P_{1}^{0}$.
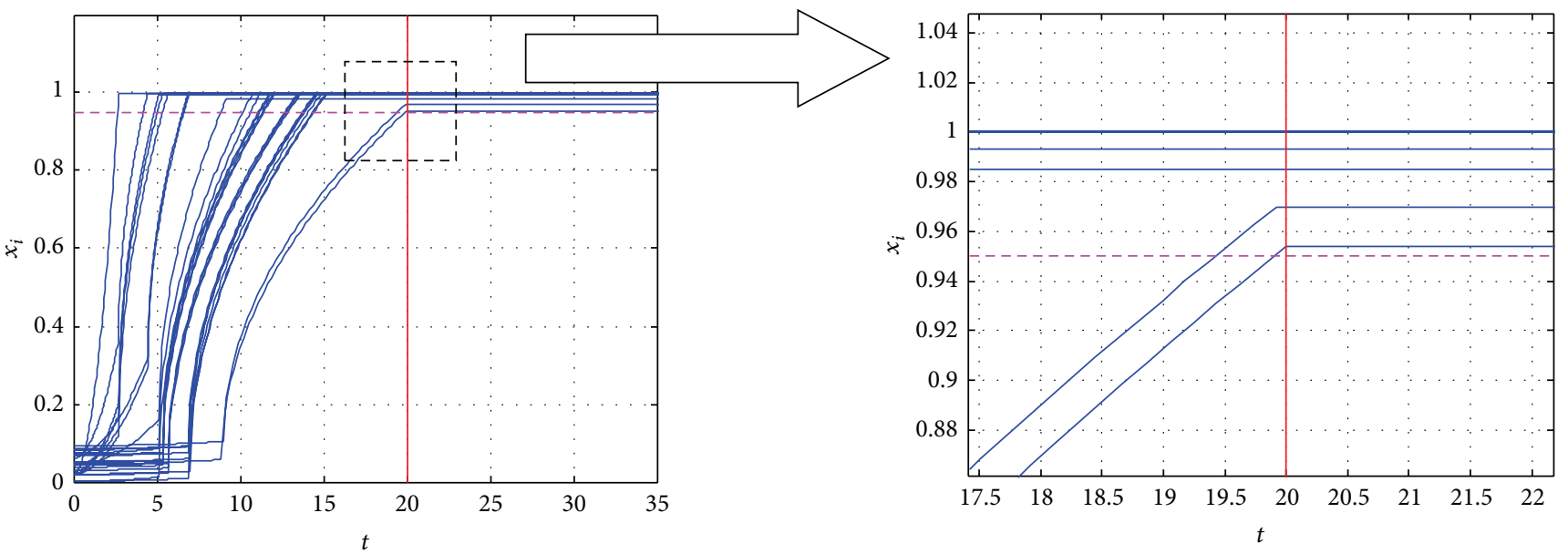

(a) The incomplete ODMS process with $U P_{1}^{1}: P_{1}^{1}$

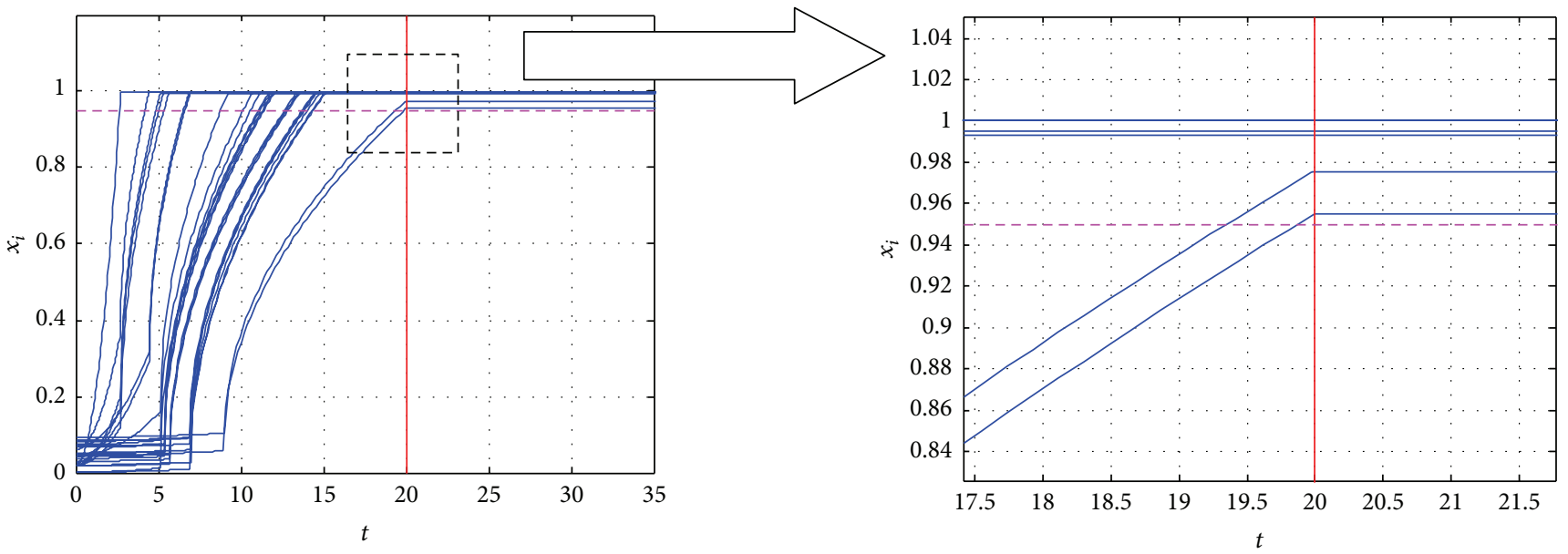

(b) The incomplete ODMS process with $U P_{1}^{2}: P_{1}^{2}$

FIGURE 13: Incomplete ODMS under stable situation. 

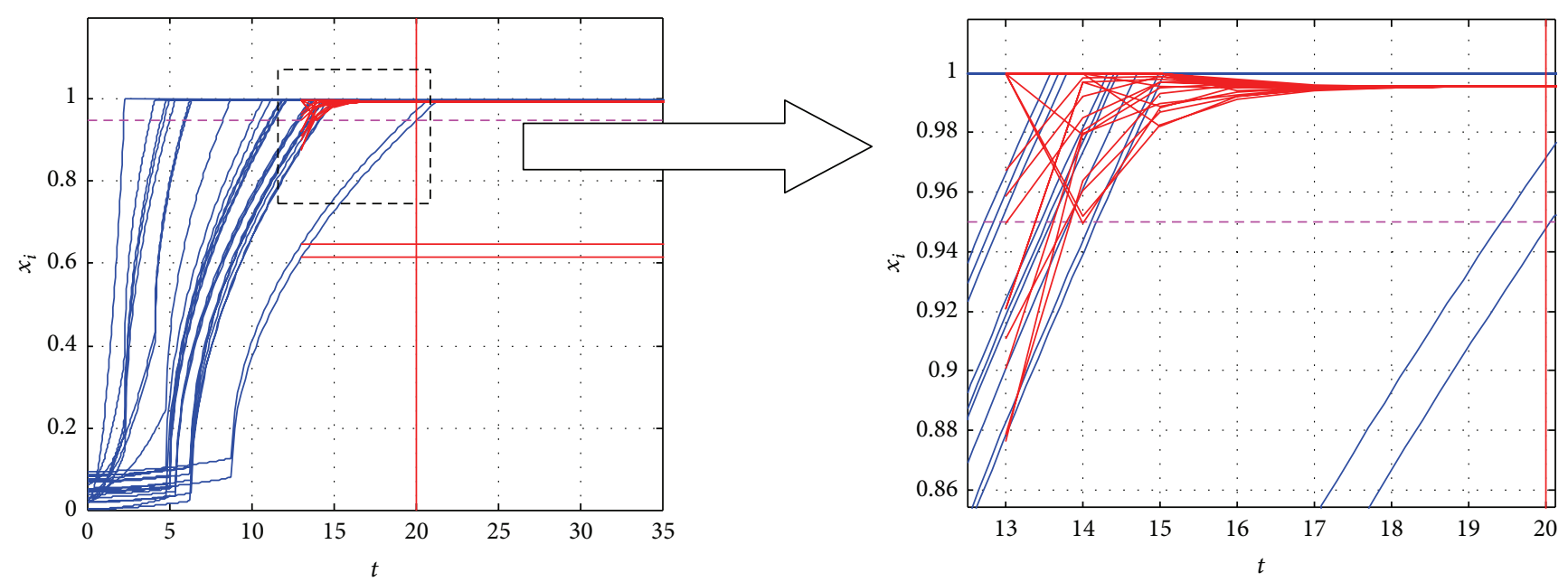

Figure 14: Comparing with the models in [5].

TABLE 9: Strategies sets of incomplete ODMS.

\begin{tabular}{|c|c|c|c|}
\hline No. & EID & $U P_{1}^{1}$ & $U P_{1}^{2}$ \\
\hline 1 & $\mathrm{o}$ & - & - \\
\hline 2 & op1 & 0.985 & 0.993 \\
\hline 3 & op2 & 1 & 1 \\
\hline 4 & op3 & 1 & 1 \\
\hline 5 & op4 & 1 & 1 \\
\hline 6 & p11 & 1 & 1 \\
\hline 7 & p12 & 1 & 1 \\
\hline 8 & p13 & 0.992 & 0.994 \\
\hline 9 & p131 & 0.996 & 0.990 \\
\hline 10 & pl32 & 0.983 & 0.987 \\
\hline 11 & $\mathrm{p} 21$ & 1 & 1 \\
\hline 12 & p211 & 1 & 1 \\
\hline 13 & p212 & 1 & 1 \\
\hline 14 & p213 & 1 & 1 \\
\hline 15 & $\mathrm{p} 22$ & 1 & 1 \\
\hline 16 & p221 & 1 & 1 \\
\hline 17 & p222 & 1 & 1 \\
\hline 18 & p223 & 1 & 1 \\
\hline 19 & p31 & 1 & 1 \\
\hline 20 & p32 & 1 & 1 \\
\hline 21 & p33 & 1 & 1 \\
\hline 22 & p34 & 1 & 1 \\
\hline 23 & $\mathrm{p} 41$ & 1 & 1 \\
\hline 24 & $\mathrm{p} 42$ & 1 & 1 \\
\hline 25 & p43 & 1 & 1 \\
\hline
\end{tabular}

After the SCL of the entities is all in the complete status, then CSSync $=1$, which reflects that complete ODMS. The process of CSSync is shown in Figure 15.

For Kuramoto model in [16], synchronization process is only controlled by a coupled coefficient (as feedback of synthesized information difference), and it does not take the strategies of ODMS into account, which makes it hard to adapt to the environment change.

According to the ODMS processes in NET1, NET2 and NET3, we can summarize four factors for influencing the ODMS capability of different organizations, including organization structure, shared information resources, interactions patterns, and allocation of decision rights. Then inhibitors and enablers for different ODMS factors are given for compared analyzing in Table 10.

For the 1st factor about organization structure, with the networked level of C2 organization increasing from NET1 to NET3, IPA of operational entities can be improved remarkably for promoting the SCL, which is benefit for enhancing the ODMS performance. Then enabler of networked structure is networked structure, and the inhibitor of organization structure is traditional tree structure. For the 2 nd factor about shared information resources, as P1 in Figure 6(a) and ODMS process in Figure 10, the situation information resources are shared in sufficiency, which is good for shortening the ST. Thus enabler for the factor is shared across members and all information accessible across entities, and inhibitor for the factor is none or a few shared (mainly kept within own entities). For the 3rd factor about interactions patterns, comparing $\mathrm{P} 5$ with $\mathrm{P} 1, \mathrm{P} 2, \mathrm{P} 3$, and $\mathrm{P} 4$, as the entity op2 is not the most important entity in NET1, there are fixed restrictions of allocating decision rights, which is not good for forming the suitable situation interaction route for adapting to the environment influence. So interactions patterns with highly fixed and constrained in superior-subordinate will inhibit ODMS capability improvement. However, compared with the complete ODMS processes in NET2 (within Figure 10) and NET3 (within Figure 12), there are several interaction patterns to be selected, and we can change situation interaction pattern in agility, which is good for improving the SIQ; thus interactions patterns with unconstrained and agile across entities can be regarded as the enabler of the factor. For the 4th factor about allocation of decision rights, the fixed taskrole-based decision rights can inhibit the situation cognition 
TABLE 10: ODMS inhibitors and enablers.

\begin{tabular}{lll}
\hline ODMS factors & Inhibitors & Enablers \\
\hline Organization structure & Traditional tree structure & $\begin{array}{l}\text { Networked structure } \\
\text { Shared across members and all }\end{array}$ \\
Shared information resources & None or a few shared (mainly kept within own entities) & $\begin{array}{l}\text { information accessible across entities. } \\
\text { infors }\end{array}$ \\
Interactions patterns & Highly fixed and constrained in up-down grade & Unconstrained and agile across entities \\
Allocation of decision rights & Fixed task-role based & Distributed to all subjects \\
\hline
\end{tabular}
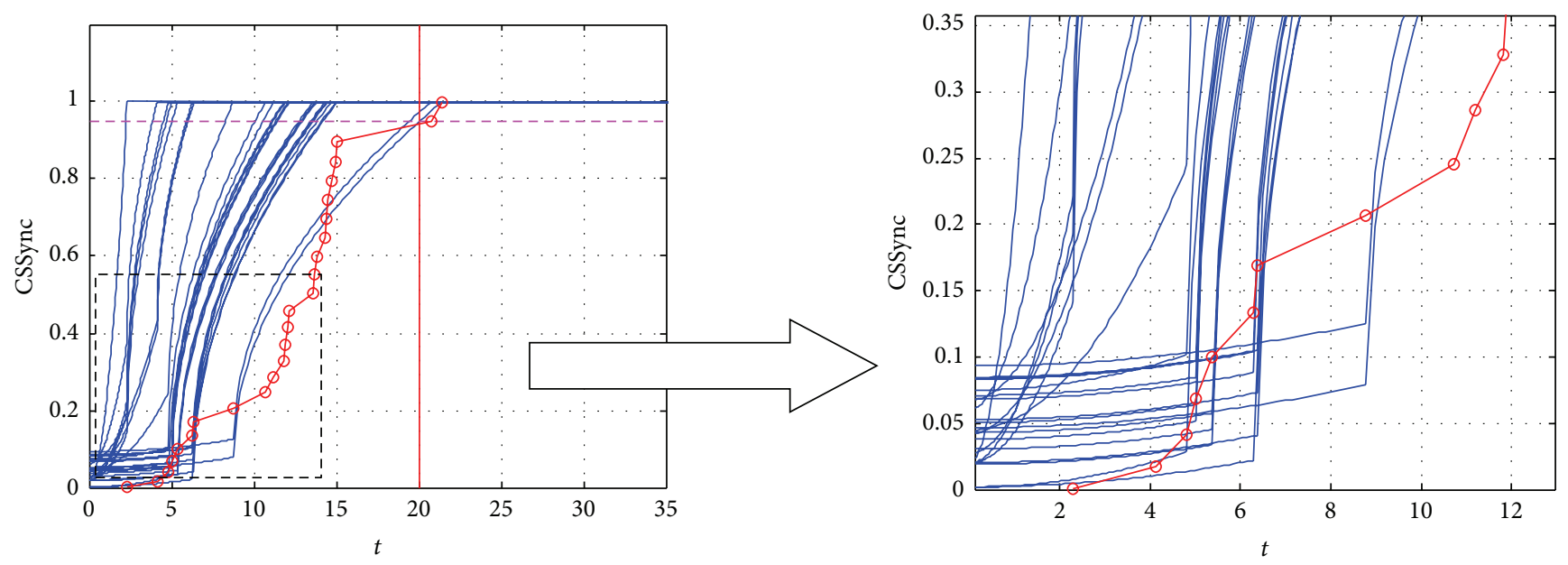

Figure 15: Comparing with the models in [13].

level formation and interaction pattern optimizing selection, which will depress the ODMS capability of the organization. However, with the networked level of organizations increasing, the decision rights can be distributed to different subjects according to situation change, which is good for adjusting situation interaction routes to adapt to environment influence and can be regarded as enabler for the factor.

\section{Conclusion and Further Work}

With the viewpoint of time consumed, the model of ODMS is constructed with three phases under complex environment, and the strategies of ODMS are put forward in the paper within environment influence and time restriction to improve the ODMS performance for adapting to environment. Concerning the several factors in ODMS, such as situation interaction patterns, situation interaction route, and situation in different changing patterns, the process of ODMS can be controlled more reasonable, and the results of this model are better than the other previous models to fulfill the actual demand.

As the environment of battlefield is complex and uncertain, the influence on ODMS is changing instantly. The C2 organization is the critical factor. So the future work might involve the dynamic self-adaptation of $\mathrm{C} 2$ organization within different environment. If several operational entities cannot percept situation information, the organization structure should be adjusted and optimized to make these entities form IPA. Then how to adjust C2 organization in optimization, such as adding and cutting down entities, or changing the structure on the basis of fixed entities, is the difficult problems to enhance the ODMS performance.

\section{Acknowledgment}

Research works in this paper are supported by the National Natural Science Foundation of China (61272011).

\section{References}

[1] D. Alberts and J. Garstka, Network Centric Warfare: Developing and Leveraging Information Superiority (2nd Edition Revised), The Command and Control Research Program (CCRP) Publication Series, Washington, DC, USA, 2002.

[2] J. R. Butler, Network Centric Warfare (NCW): The Mechanism for Change, ADA520230, Marine Corps University, 2003.

[3] J. Garstka and D. Alberts, Network Centric Operations Conceptual Framework (Version 2.0), Evidence Based Research, 2007.

[4] H. Liu and L. Zhang, "Analysis on opinion emergence of group communication," Complex Systems and Complexity Science, vol. 4, no. 1, pp. 45-52, 2004.

[5] B. Wang, Research on Operation Synchronization Modeling Based on Complex Networks, National University of Defense Technology, 2007.

[6] X. K. Bao, Modeling and Analyzing of Networked Operation Synchronization, National University of Defense Technology, 2009.

[7] S. S. Fatima, M. Wooldridge, and N. R. Jennings, "An agendabased framework for multi-issue negotiation," Artificial Intelligence, vol. 152, no. 1, pp. 1-45, 2004. 
[8] J. Scrocca, M. Molz, and A. Kott, "Collaborative awareness: experiments with tools for battle command," in Proceedings of the 11th International Command and Control Research and Technology Symposium, 2006.

[9] A. Kirlik and R. Strauss, "Situation awareness as judgment I: statistical modeling and quantitative measurement," International Journal of Industrial Ergonomics, vol. 36, no. 5, pp. 463-474, 2006.

[10] R. Strauss and A. Kirlik, "Situation awareness as judgment II: experimental demonstration," International Journal of Industrial Ergonomics, vol. 36, no. 5, pp. 475-484, 2006.

[11] A. Kirlik and R. Strauss, "Situation awareness as judgment I: statistical modeling and quantitative measurement," International Journal of Industrial Ergonomics, vol. 36, no. 5, pp. 463-474, 2006.

[12] H. Meng and D. G. Zhang, "Model of situation awareness velocity under continuous changing situation," Systems Engineering and Electronics, vol. 31, no. 12, pp. 2909-2913, 2009.

[13] M. Manso and J. Moffat, "Defining and measuring cognitiveentropy and cognitive self-synchronization," in Proceedings of the International Command and Control Research and Technology Symposium, 2011.

[14] M. Ruddy, "ELICIT-the experimental laboratory for investigating collaboration, information sharing and trust," in Proceedings of the International Command and Control Research and Technology Symposium, 2007.

[15] A. H. Dekker, "Average distance as predictor of synchronisability in networks of coupled oscillators," in Proceedings of the 33rd Australasian Computer Science Conference, vol. 102, pp. 127-131, 2010.

[16] A. H. Dekker, "Analyzing C2 structures and self-synchronization with simple computational models," in Proceedings of the International Command and Control Research and Technology Symposium, 2011.

[17] L. J. Pan, X. J. Li, and D. S. Hao, "Research on modeling of battle collaboration effect," Journal of System Simulation, vol. 23, no. 12, pp. 2561-2565, 2011.

[18] P. Holme, "Congestion and centrality in traffic flow on complex networks," Advances in Complex Systems, vol. 32, no. 6, pp. 163$176,2003$.

[19] D. Gómez, E. González-Arangüena, C. Manuel, G. Owen, M. del Pozo, and J. Tejada, "Centrality and power in social networks: a game theoretic approach," Mathematical Social Sciences, vol. 46, no. 1, pp. 27-54, 2003.

[20] S. A. David and M. James, Network Centric Warfare and Complexity Theory, Publishing House of Electronics Industry, Beijing, China, 2004.

[21] Y. Liu, J. Zhao, J. N. Liu, D. J. Feng, and G. Y. Wang, "Novel battlefield electromagnetic environment classification method based on combat efficacy of weapon systems," Systems Engineering and Electronics, vol. 33, no. 5, pp. 1059-1062, 2011.

[22] X. C. Gong, Y. C. Jiang, and H. Chen, "Command and control in the network-centric warfare," Fire Control \& Command, vol. 35, no. 3, pp. 1-8, 2010.

[23] J. Wu and Y. J. Tan, "Finding the most vital node by node contraction in communication networks," in Proceedings of the International Conference on Communications, Circuits and Systems, vol. 2, pp. 1283-1286, May 2005.

[24] Y. Zhao, Z. Wang, J. Zheng, and X. Guo, "Finding most vital node by node importance contribution matrix in communication networks," Journal of Beijing University of Aeronautics and Astronautics, vol. 35, no. 9, pp. 1076-1079, 2009.
[25] J. Jiang, Research on Executable Architecture and the Executable Method of DoDAF, National University of Defense Technology, 2008. 


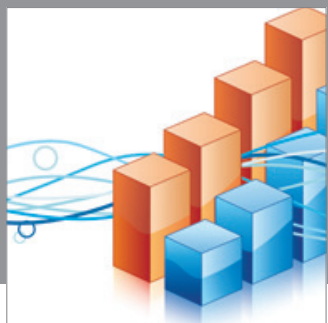

Advances in

Operations Research

mansans

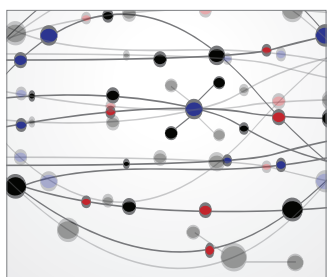

The Scientific World Journal
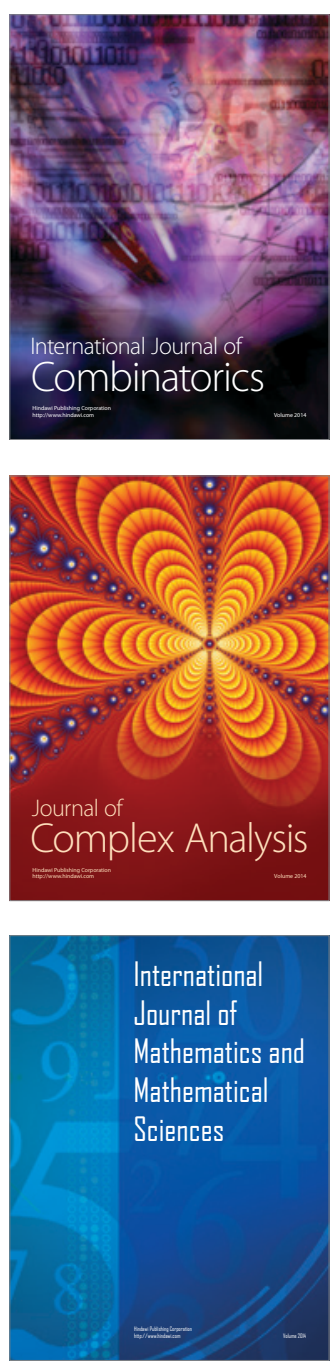
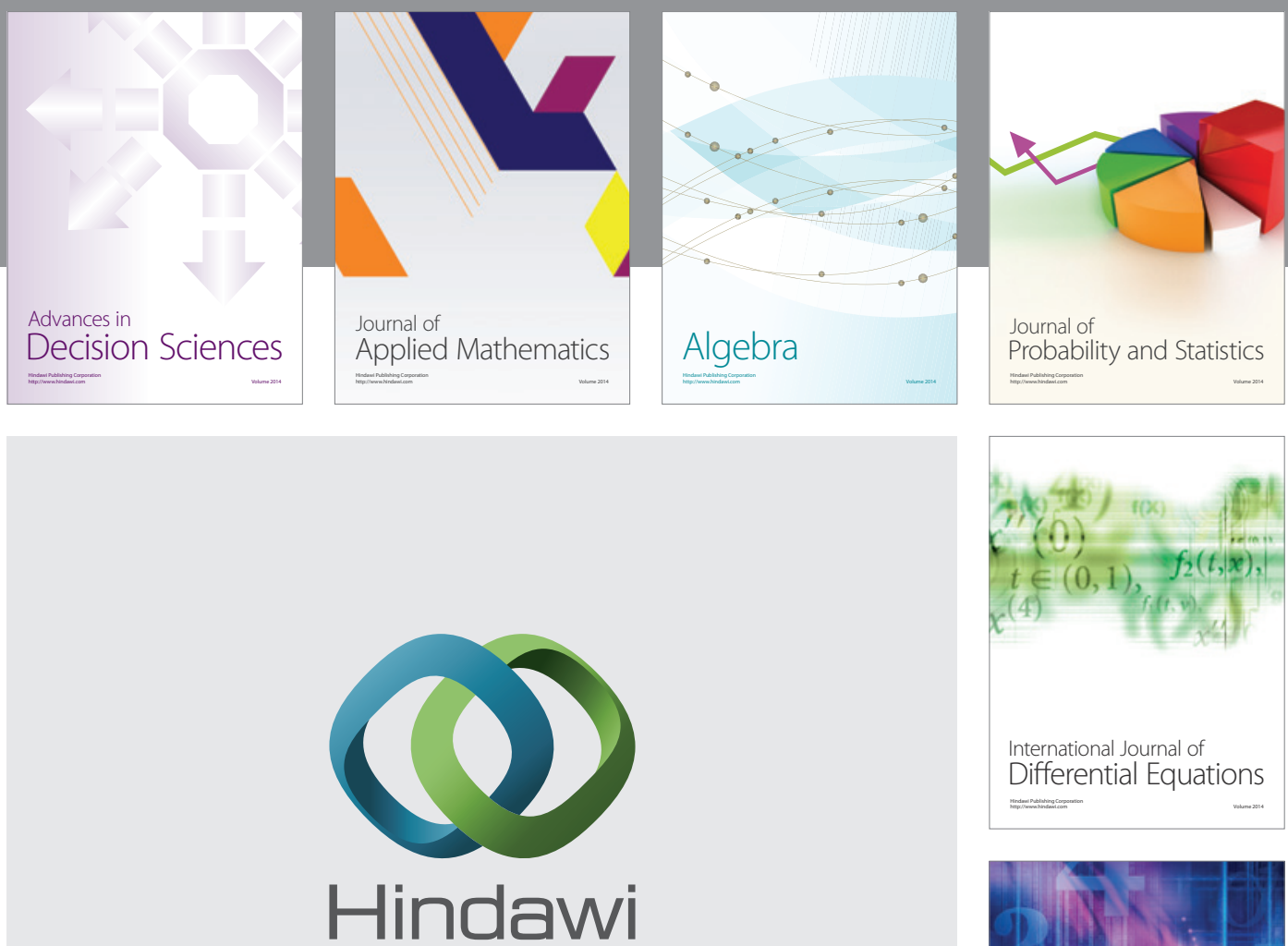

Submit your manuscripts at http://www.hindawi.com
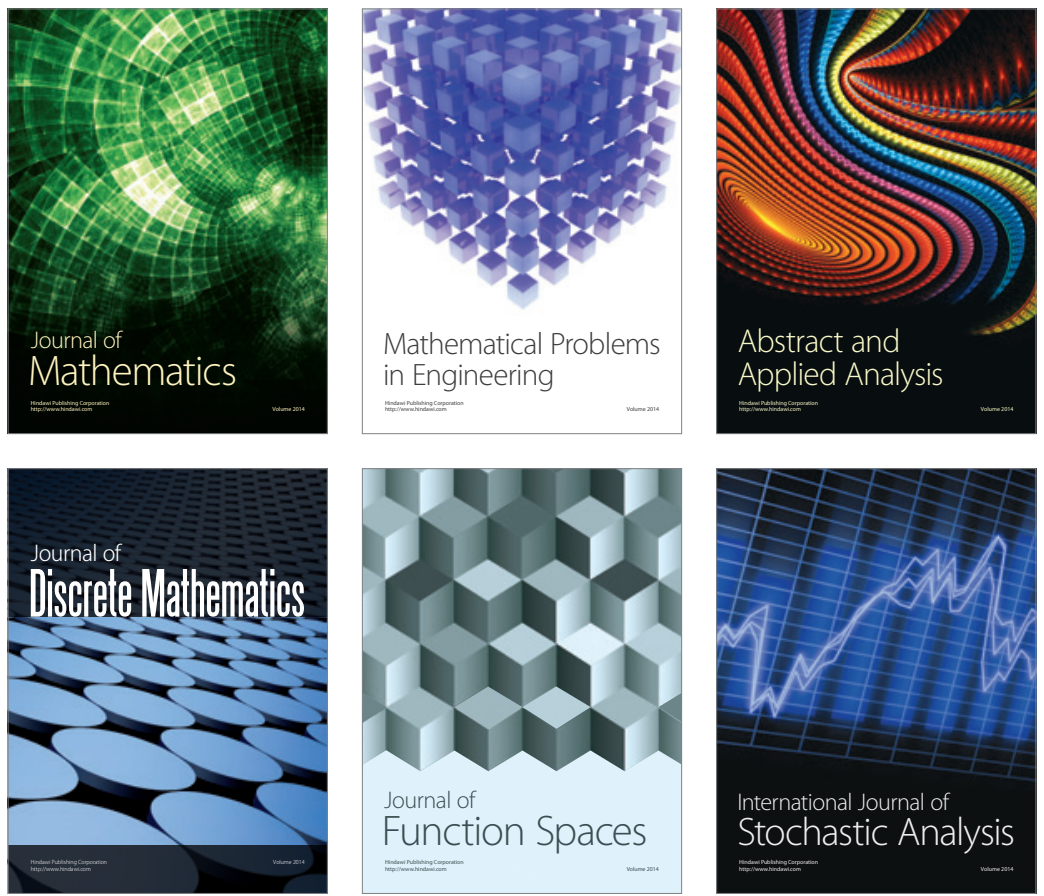

Journal of

Function Spaces

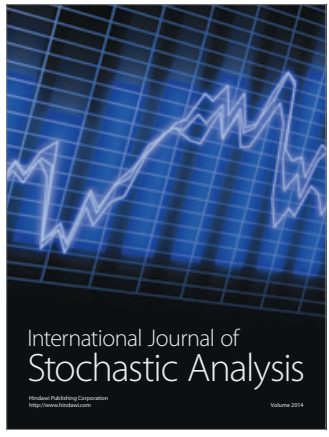

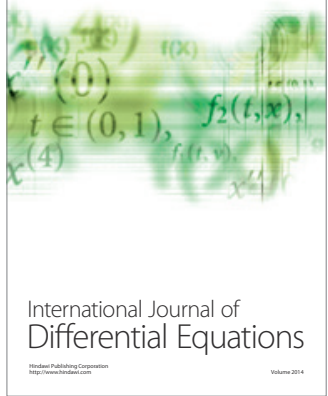
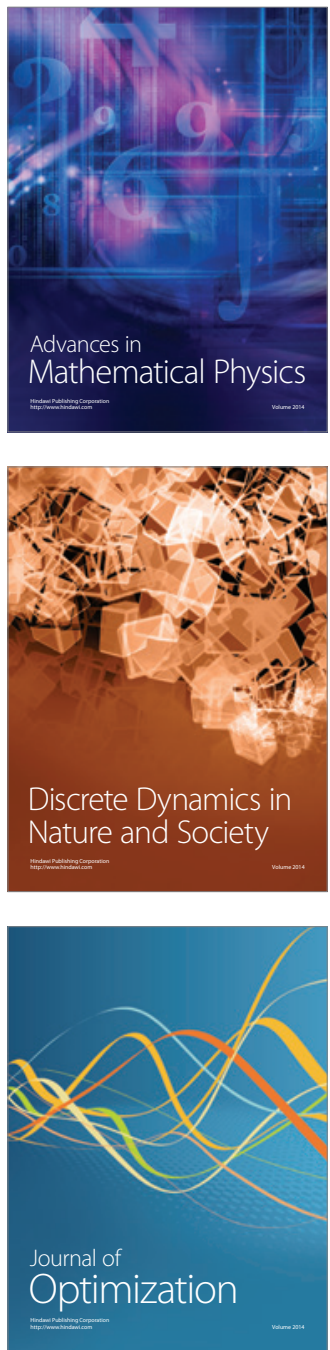